\title{
Oxidized CaMKII promotes asthma through the activation of mast cells
}

\author{
Jingjing Qu, ${ }^{1,2}$ Danh C. Do, ${ }^{1}$ Yufeng Zhou, ${ }^{1,3}$ Elizabeth Luczak, ${ }^{4}$ Wayne Mitzner, ${ }^{5}$ Mark E. Anderson, ${ }^{4}$ \\ and Peisong Gao ${ }^{1}$ \\ 'Division of Allergy and Clinical Immunology, Johns Hopkins School of Medicine, Baltimore, Maryland, USA. \\ ${ }^{2}$ Department of Respiratory Medicine, Xiangya Hospital, Central South University, Changsha, Hunan, China. \\ ${ }^{3}$ Institute of Biomedical Sciences and Children's Hospital, Fudan University, Shanghai, China; Key Laboratory \\ of Neonatal Diseases, Ministry of Health, Shanghai, China. ${ }^{4}$ Department of Medicine, Johns Hopkins School of Medicine, \\ Baltimore, Maryland, USA. ${ }^{5}$ Department of Environmental Health Sciences, Johns Hopkins Bloomberg School of Public \\ Health, Baltimore, Maryland, USA.
}

Oxidation of calmodulin-dependent protein kinase II (ox-CaMKII) by ROS has been associated with asthma. However, the contribution of ox-CaMKII to the development of asthma remains to be fully characterized. Here, we tested the effect of ox-CaMKII on IgE-mediated mast cell activation in an allergen-induced mouse model of asthma using oxidant-resistant CaMKII MMVV $\delta$ knockin

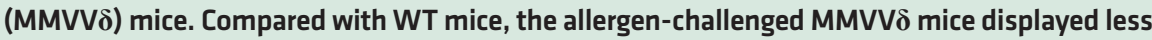
airway hyperresponsiveness (AHR) and inflammation. These MMVV $\delta$ mice exhibited reduced levels of ROS and diminished recruitment of mast cells to the lungs. OVA-activated bone marrowderived mast cells (BMMCs) from MMVV $\delta$ mice showed a significant inhibition of ROS and oxCaMKII expression. ROS generation was dependent on intracellular $\mathrm{Ca}^{2+}$ concentration in BMMCs. Importantly, OVA-activated MMVV $\delta$ BMMCs had suppressed degranulation, histamine release, leukotriene C4, and IL-13 expression. Adoptive transfer of WT, but not MMVVס, BMMCs, reversed the alleviated AHR and inflammation in allergen-challenged MMVV $\delta$ mice. The CaMKII inhibitor KN-93 significantly suppressed IgE-mediated mast cell activation and asthma. These studies support a critical but previously unrecognized role of ox-CaMKII in mast cells that promotes asthma and suggest that therapies to reduce ox-CaMKII may be a novel approach for asthma.

Conflict of interest: M.E. Anderson is a cofounder of Allosteros Therapeutics, a biotech company aiming to develop CaMKII-based therapies.

Submitted: August 16, 2016 Accepted: November 29, 2016 Published: January 12, 2017

Reference information: JCI Insight. 2017;2(1):e90139. doi:10.1172/ji.insight.90139.

\section{Introduction}

ROS are an important mediator in allergic diseases and asthma (1-5), but clear understanding of the molecular pathways disrupted by ROS is lacking. Exposure of the airway epithelium to environmental pollutants or allergens is known to induce oxidative stress either directly or through the induction of local inflammatory processes that lead to the secondary production of ROS (6-8). Previous studies suggest that the multifunctional $\mathrm{Ca}^{2+} /$ calmodulin-dependent protein kinase II (CaMKII) is within one of the downstream signaling pathways activated by ROS (9). CaMKII has four isoforms, $\alpha, \beta, \delta$, and $\gamma$, encoded by different genes, displaying distinct but overlapping expression patterns (10). Both the $\alpha$ and $\beta$ isoforms are almost exclusively expressed in the brain, whereas the $\delta$ and $\gamma$ isoforms are expressed more ubiquitously. Of these, CaMKII $\delta$ in airway smooth muscle has been shown to promote allergen-induced airway hyperresponsiveness (AHR) and inflammation (11). CaMKII is held in an inactive state but can be activated by oxidization at methionines $281 / 282$ in the CaMKII regulatory domain in the presence of ROS $(12,13)$, locking the oxidized CaMKII (ox-CaMKII) into a persistently active configuration. Both NADPH oxidase (12-14) and mitochondria $(15,16)$ are considered as major sources of ROS for ox-CaMKII (12). Ox-CaMKII has been linked with various diseases, including vascular disease $(14,17)$, diabetes (15), asthma (18), and cancer (16), and has been shown to promote inflammatory signaling (19), cell proliferation (20), and ion channel activity (21). Interestingly, increased expression of ox-CaMKII has been observed in the airway epithelium of asthmatic patients, which was correlated with the severity of asthma (18). Thus, CaMKII may serve as a critical ROS sensor and a candidate target for asthma therapy.

Mast cells are known to be critical in the regulation of allergic diseases, in part because of their preferential localization at the site of the tissue mucosa where coexposure of antigens and environmental 
chemicals often occurs (22). The IgE receptor FceRI-dependent pathway in mast cells is the predominant pathway contributing to various pathophysiological events in acute and chronic inflammation (23-25). Mast cells also express additional receptors, including pattern recognition receptors (e.g., TLRs), aryl hydrocarbon receptor (AhR) (26), and complement receptors to sense environmental stimuli (27). Mast cell-deficient $\left(\mathrm{Kit}^{\mathrm{W}-\mathrm{sh} / \mathrm{W}-\mathrm{sh}}\right)$ mice exhibited an exacerbated protease-induced lung inflammation associated with reduction in lung Tregs, suggesting that mast cells are critical in allergen-induced lung inflammation and $\mathrm{T}$ cell differentiation (28). Human lung mast cells are associated with airway smooth muscle bundles in patients with allergic asthma and have been linked to airway inflammation, tissue remodeling, airway smooth muscle $\beta 2$ adrenoceptor activation, and AHR (22, 29-31). Considering the critical role of ox-CaMKII in inflammatory signaling (19), we hypothesized that exposure to environmental allergens may cause irreversible oxidative modifications of CaMKII, which may regulate mast cell function and lead to the development of allergic diseases and asthma.

In this study, we provide clear evidence that loss of ox-CaMKII prevents environmental allergen-induced AHR, lung inflammation, and Th2 cytokine production using newly generated oxidant-resistant

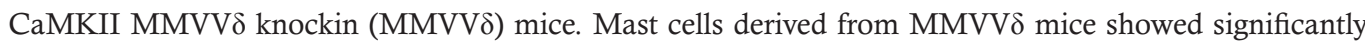
less ROS and reduced IgE-mediated mast cell activation, including degranulation, histamine release, and leukotriene C4 (LTC4) production and IL-13 production, and anaphylactic responses (passive cutaneous anaphylaxis [PCA]) compared with WT littermate controls. Importantly, adoptive transfer of WT bone marrow-derived mast cells (BMMCs), but not MMVV $\delta$ mast cells, reversed the preventive role of MMVV $\delta$ in cockroach allergen-induced (CRE-induced) AHR, lung inflammation, and Th2 cytokine production in MMVV $\delta$ mice. Furthermore, we demonstrated that inhibition of CaMKII prevented IgE-mediated mast cell activation and development of asthma. Collectively, these studies suggest a conceptual framework for the role of ROS in asthma by linking the environmental allergen exposure-ROS-ox-CaMKII axis to mast cell activation and the development of asthma and potentially other allergic diseases.

\section{Results}

ox-CaMKII regulates CRE-induced lung function and inflammation. We first asked about the expression profile of CaMKII isoforms in mouse lung tissues. We found that CaMKII $\delta$ and CaMKII $\gamma$, but not CaMKII $\alpha$ and CaMKII $\beta$ (Supplemental Figure 1A; supplemental material available online with this article; doi:10.1172/jci. insight.90139DS1), were expressed in the mouse lung tissues (Supplemental Figure 1A) and BMMCs (Supplemental Figure 1B), and there was no difference in $\delta$ and $\gamma$ isoform expression in BMMCs from WT or MMVV $\delta$ mice. To better understand the role of CaMKII $\delta$, we generated the oxidant-resistant CaMKII MMVV knockin

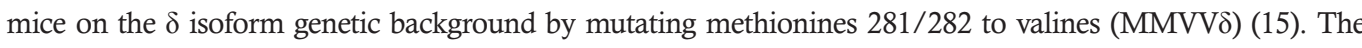
MMVV mutation selectively eliminates ROS activation without affecting $\mathrm{Ca}^{2+}$ and calmodulin-dependent $\mathrm{CaM}$ KII activation (12). To understand the role of the ROS-resistant MMVV $\delta$ in asthma, we used an established CRE-induced mouse model with either WT or MMVV $\delta$ mice (Figure 1A). CRE-challenged WT and MMVV $\delta$ mouse lungs showed increased inflammatory cell infiltration with goblet cell hyperplasia, as assessed by H\&E and PAS staining (Figure 1B). In contrast, lungs from PBS control mice showed normal or undetectable airway inflammation after CRE sensitization and challenge. Compared with WT mice, CRE-challenged MMVV $\delta$ mice showed less airway inflammation and goblet cell hyperplasia. When we analyzed lung function, CRE-sensitized WT mice showed physiological changes consistent with asthma, including increased airway resistance (Figure 1C) and diminished airway compliance (Figure 1D), but there were significantly fewer physiological changes in CRE-exposed MMVV $\delta$ mice than in CRE-exposed WT mice. Moreover, we found a significant reduction in the total number of inflammatory cells (Figure 1E), especially eosinophils, lymphocytes, and neutrophils among all tested cell types, in bronchoalveolar lavage (BAL) fluids of CRE-challenged MMVV $\delta$ mice when compared with WT mice (Figure 1F). These MMVV $\delta$ mice also produced lower serum titers of CRE-specific IgE and IgG1 than WT counterparts (Figure 1G). Allergic airway inflammation involves Th2 responses in the lungs $(32,33)$. We found that CRE-treated MMVV $\delta$ mice showed much lower concentrations of Th2 cytokines IL-4, IL-5, and IL-13 (Figure 1H). In contrast, these MMVV $\delta$ mice showed higher levels of IFN- $\gamma$ in BAL fluid compared with WT mice (Supplemental Figure 2A), but no changes were observed for IL-12 (Supplemental Figure 2B). To further examine whether these findings can be applied to other allergens, we generated a house dust mite-induced (HDM-induced) mouse model of asthma following the same protocol as CRE. Similar results were obtained as CRE (Supplemental Figure 3, A-F). Taken together, these data suggest that oxidative activation of CaMKII plays a critical role in environmental allergen-induced AHR and inflammation. 
A

CRE or PBS (Sensitization, IT)

CRE or PBS

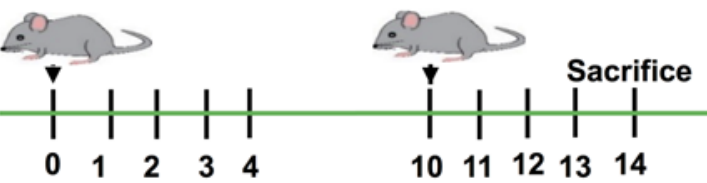

Time (days)
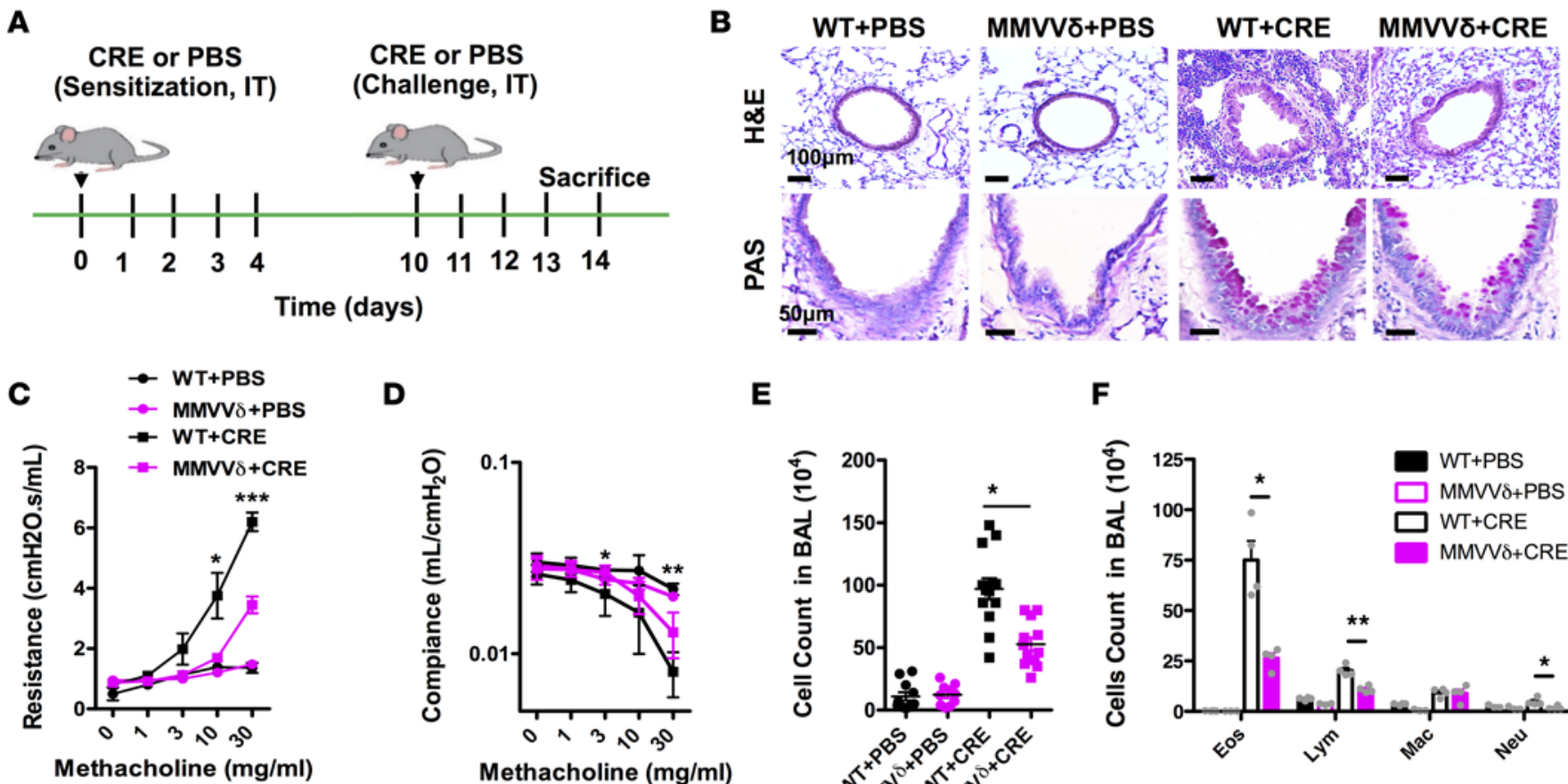

D

E

F
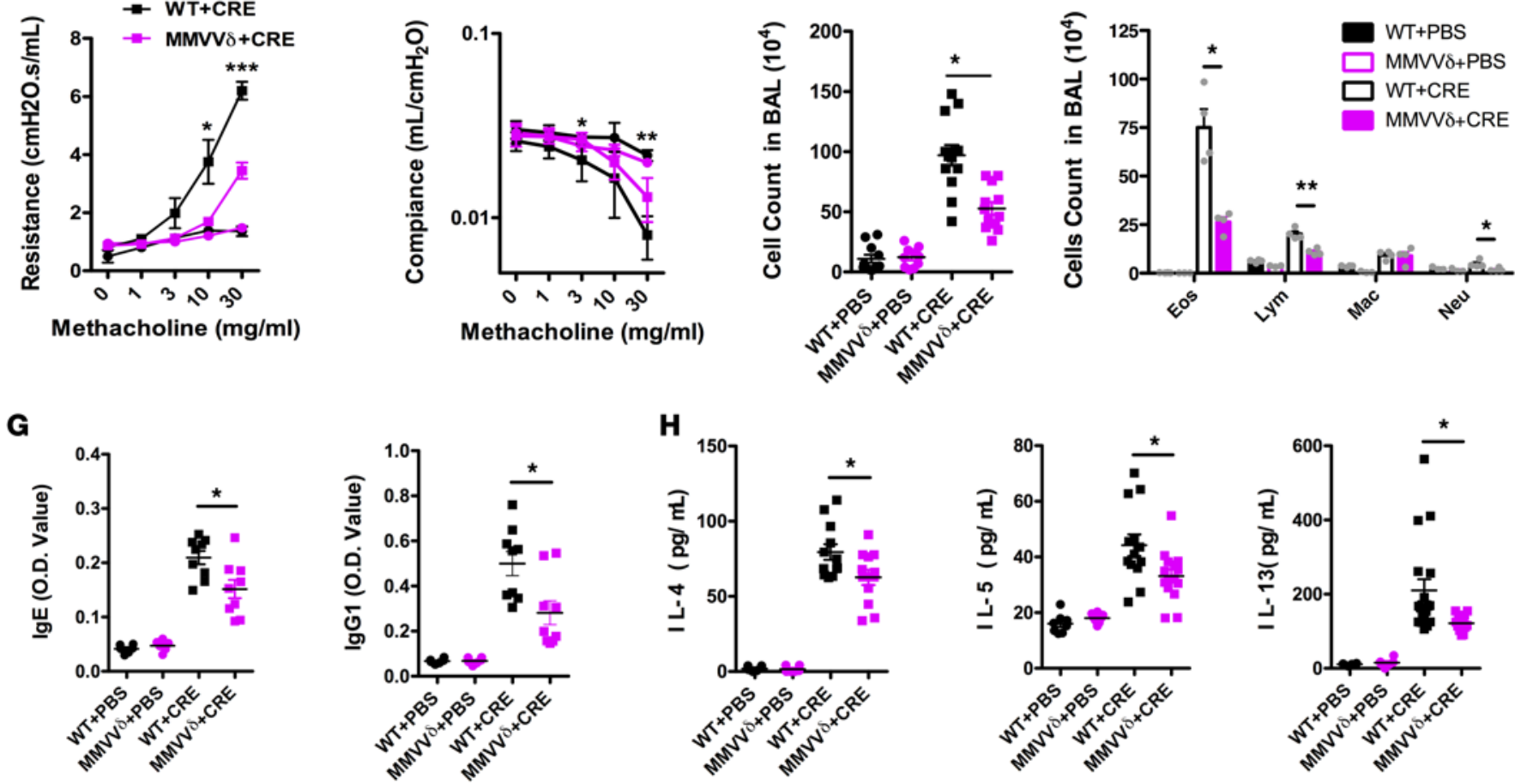

Figure 1. Ox-CaMKII regulates cockroach allergen-induced airway hyperresponsiveness and inflammation. (A) Protocol for cockroach allergen-induced (CRE-induced) mouse model of asthma. (B) Paraffin tissue sections of lung were stained with H\&E (top; scale bar: $100 \mu \mathrm{m}$ ) and periodic acid-Schiff (PAS, bottom; scale bar: $50 \mu \mathrm{m}$ ). (C and $\mathbf{D})$ Lung resistance (C) and compliance (D) in response to increasing concentrations of methacholine using the forced oscillation technique (FlexiVent, SCIREQ) from CRE-challenged WT $(n=6)$ and MMVV $\delta(n=6)$ mice. (E and F) Bronchoalveolar lavage $(B A L)$ total $(\mathbf{E})$ and differential cell counts (F) of PBS- $(n=7)$ and CRE-challenged WT $(n=11)$ and MMVV $\delta(n=11)$ mice. (C) Serum levels of cockroach allergen-specific IgE and IgG1. (H) Levels of cytokines in BAL. Data represent mean \pm SEM; comparisons were made using 2-tailed Student's $t$ test between CRE-treated WT vs. MMVV $\delta .{ }^{*} P<0.05,{ }^{* *} P<0.01,{ }^{* * *} P<0.001$.

ROS-resistant CaMKII MMVVS mice prevent ROS production and oxidative activity of CaMKII. Extensive studies have suggested that ROS is an important mediator in allergic diseases and asthma (1-4) To determine whether CRE can induce ROS, we detected ROS in the lung tissues of a CRE-induced mouse model by the ROS fluorescent indicator DHE (14). Compared with PBS-treated mice, increased ROS levels were noted in CRE-sensitized and challenged mice (Figure 2A). Furthermore, CRE-treated MMVV $\delta$ mice showed significantly lower levels of ROS compare with WT mice. We next detected ox-CaMKII expression in the lung tissues of a CRE-induced mouse model (Figure 2B). Homogenized lung tissues from CRE-treated mice showed significantly increased ox-CaMKII levels over those from untreated mice. Compared with WT mice, lung tissues from MMVV $\delta$ mice showed much less expression of ox-CaMKII. In contrast, total CaMKII was not significantly different among all these groups. We asked if the isoform expression profile of CaMKII was affected by CRE and found that CaMKIID (Supplemental Figure 4A), but not CaMKII $\gamma$ (Supplemental Figure 4B), was significantly expressed in lung tissues after CRE challenge. Intriguingly, CRE-induced increases in CaMKII $\delta$ mRNA were inhibited in 
A

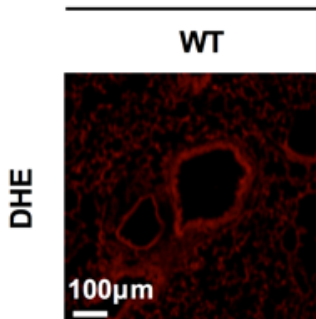

PBS



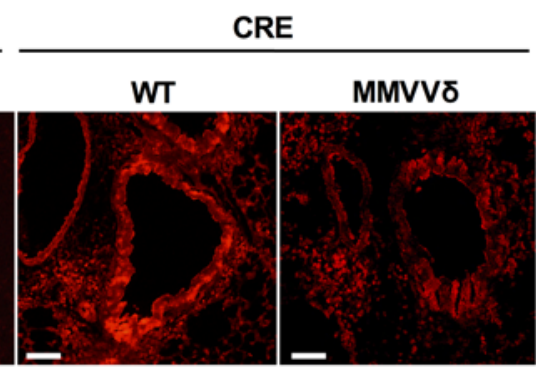

B

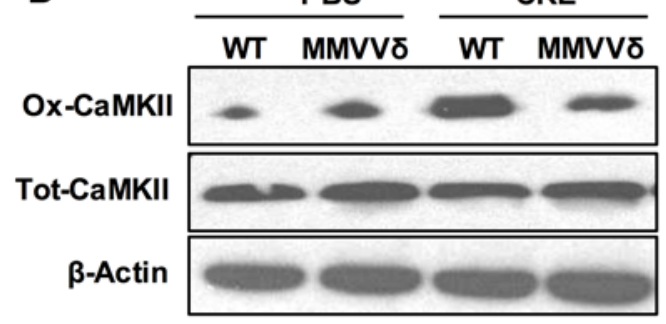

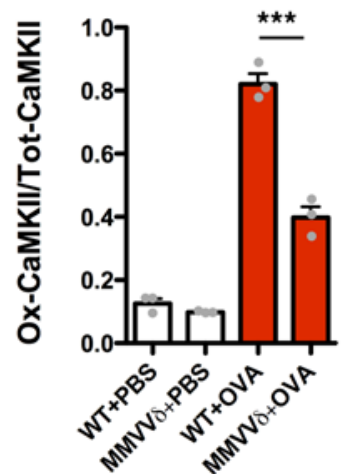

Figure 2. ROS-resistant CaMKII MMVV $\delta$ mice prevent ROS production and oxidative activity of CaMKII. (A) Representative images of dihydroethidium-stained (DHE-stained) airways of PBS- or CRE-challenged WT and MMVV $\delta$ mice. Scale bar: $100 \mu \mathrm{m}$ (B) Representative immunoblot of ox-CaMKII and total CaMKII (Tot-CaMKII) in the homogenized lung tissues of PBS- or CRE-challenged WT and MMVV $\delta$ mice. Data represent mean \pm SEM, 3 mice per group. Comparisons were made using 2-tailed Student's $t$ test between CRE-treated WT vs. MMVV. ${ }^{* *} P<0.01,{ }^{* * *} P<0.001$.

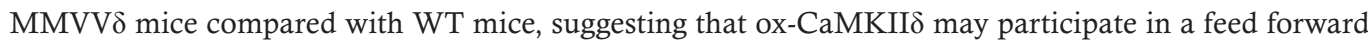
transcriptional pathway in lung. Taken together, these studies suggest that both ROS and ox-CaMKII are significantly reduced in CaMKII MMVV $\delta$ mice after exposure to CRE.

Reduced mast cell numbers in lung tissues of CRA-treated MMVVS mice. There is compelling evidence that mast cells play a key role in the pathophysiology and pathogenesis of asthma $(22,34,35)$, but the potential for mast cell activation to be affected by ROS and/or ox-CaMKII is unexplored. To test for a role of mast cells in our model, we quantified mast cell numbers in the lung tissues of CRE-exposed mice. Consistent with previous reports $(36,37)$, CRE-treated mice had a significant infiltration of mast cells in the lung tissues by immunofluorescent (Figure 3, A and C) and DAB staining for tryptase (Figure $3, \mathrm{~B}$ and D). Interestingly, the numbers of mast cells were significantly reduced in CRE-challenged MMVV $\delta$ mice compared with WT mice. Furthermore, as shown in Figure 3E, compared with WT mice, CRE-challenged MMVV $\delta$ mice showed many fewer mast cells, as identified by c-Kit staining (Figure $3 \mathrm{~F}$ ), and decreased ox-CaMKII expression in lung mast cells, as determined by ox-CaMKII ${ }^{+} \mathrm{c}-\mathrm{Kit}^{+}$staining (Figure 3G). These findings show that mast cells respond to CRE challenge and suggest ox-CaMKII may regulate mast cell migration and activation with the potential to promote asthma.

Reduced ROS levels in mast cells of ROS-resistant MMVVS mice. Given the role of mast cells in asthma, we examined the role of ox-CaMKII in ROS production in allergen-activated mast cells. BMMCs were isolated from WT and MMVV $\delta$ mice and identified by flow cytometry designed to detect c-Kit and FceRI (Figure 4A). These BMMCs are also CD49b- (data not shown). To better understand the role of CaMKIII in mast cells, we first identified the location of CaMKIII in the BMMCs by immunostaining and confocal microscopy. We found that a substantial fraction of CaMKII $\delta$ is located in mitochondria of the BMMCs (Figure 4B). We next determined whether allergen can induce oxidative activation of CaMKII in mast cells, following the protocol in Figure 4C. BMMCs were sensitized with an OVA-specific IgE monoclonal antibody (E-C1) for 16 hours, followed by crosslinkage of surface-bound IgE with OVA or PBS for 30 minutes (Figure 4C). We found increased oxidative activation of CaMKII in OVA-activated mast cells (Figure 4D). However, compared with WT cells, MMVVס mast 
A

A $\quad$ WT + PBS
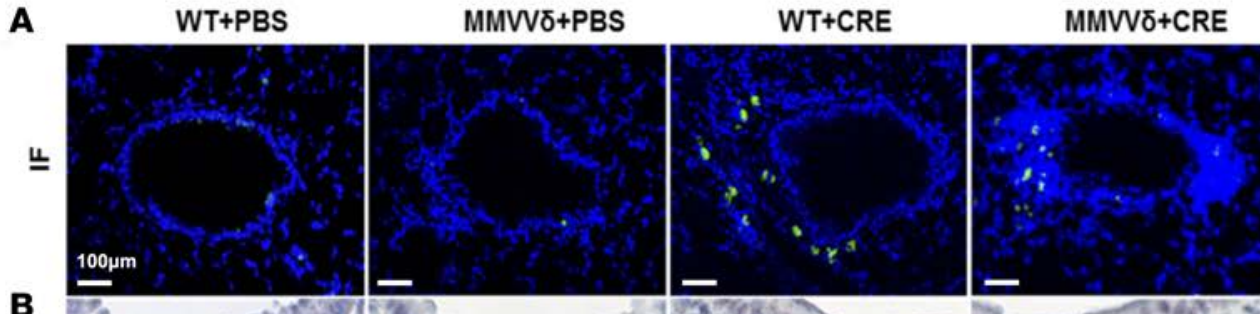

$\stackrel{\infty}{\varsigma}$
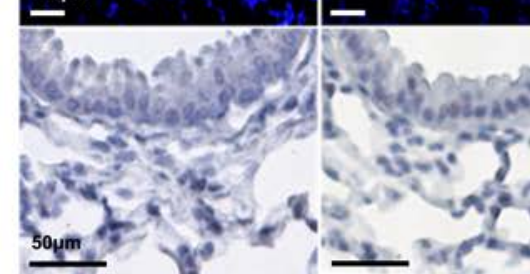
-

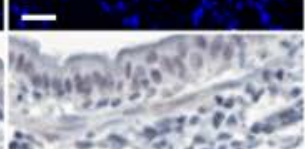

C

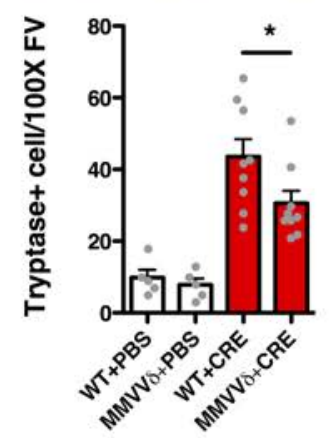

E
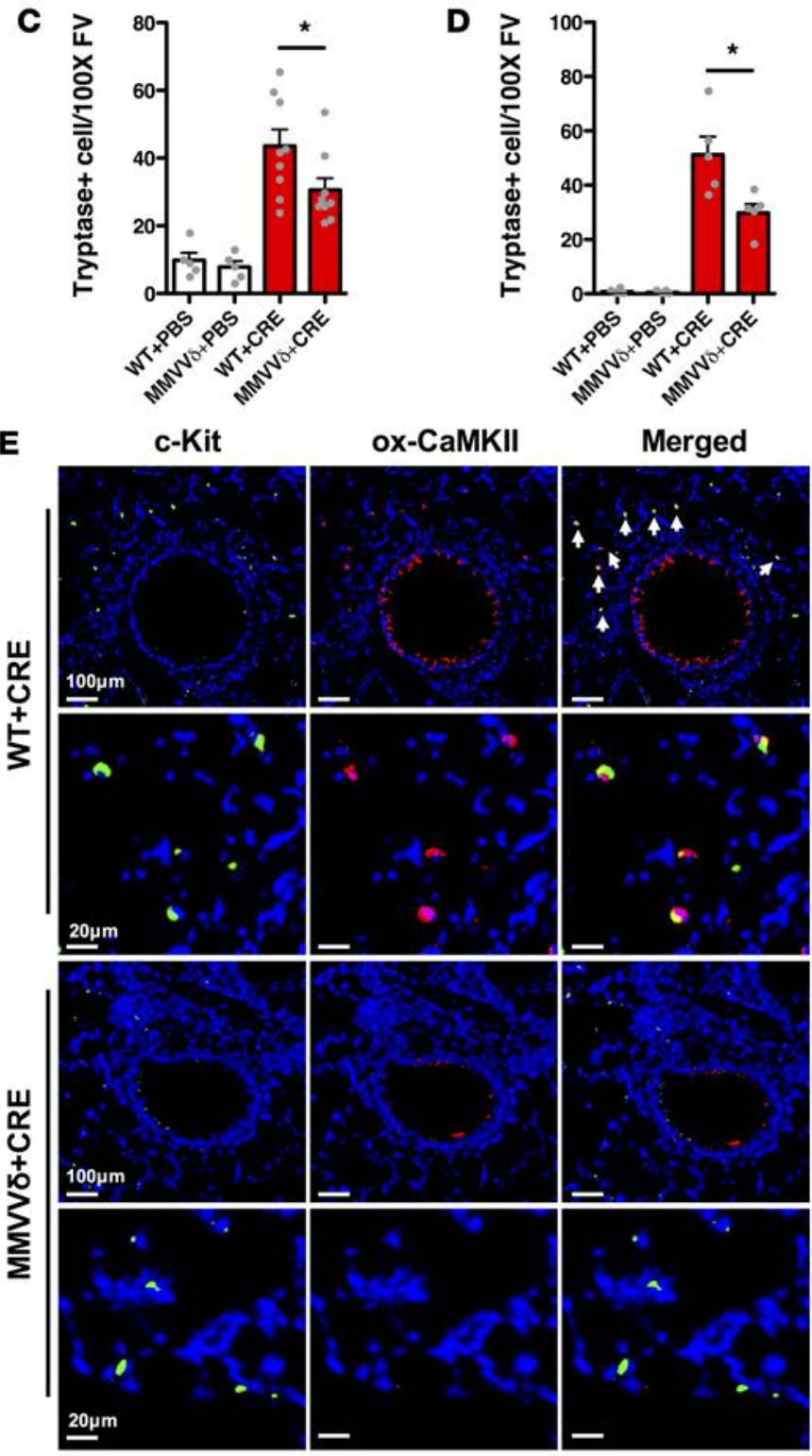

Merged
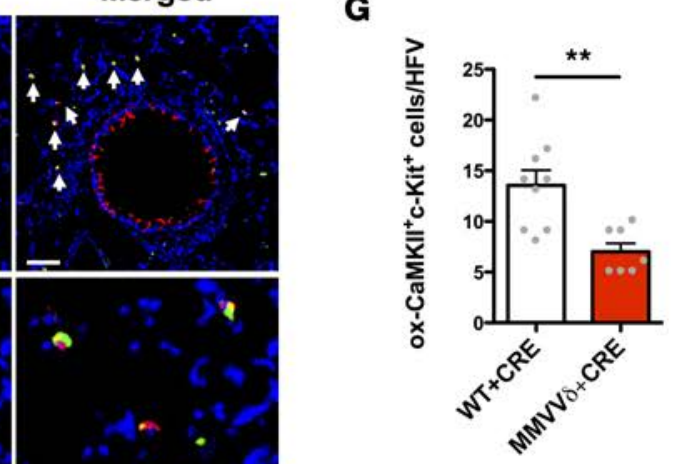

Figure 3. Reduced mast cell numbers in lung tissues of CRE-treated MMVV $\delta$ mice. (A and B) Representative images of tryptase immunofluorescence (scale bar: $100 \mu \mathrm{m}$ ) and DAB staining (scale bar: $50 \mu \mathrm{m}$ ) in lung tissue sections of PBS- or CRE-challenged $W T$ and MMVV $\delta$ mice, 3 mice per group. (C and $\mathbf{D}$ ) Quantitative data for immunofluorescence (C) and $\mathrm{DAB}$ staining (D). Mean $\pm \mathrm{SEM}, n=$ 5-9 high power field view (HFV) per group. (E) Representative images of coimmunofluorescence staining with c-Kit and ox-CaMKII in the lung tissues of CRE-challenged WT and $M M V V \delta$ mice, 3 mice per group. Scale bar: $100 \mu \mathrm{m}$ (first and third row); $20 \mu \mathrm{m}$ (second and fourth row). Quantitative data for c-Kit ${ }^{+}$ cells (F) and ox-CaMKII+c-Kit cells (C). Data represent mean \pm SEM, $n$ = 7-9 HFV per group. Comparisons were made using 2-tailed Student's $t$ test between CRE-treated WT vs. MMVV $\delta .{ }^{*} P<0.05,{ }^{* *} P<0.01$.

cells showed a significant reduction in the expression of ox-CaM$\mathrm{KII}$ (Figure 4E). In contrast, total CaMKII was not significantly different among all these groups (Figure 4F). As controls, we measured ox-CaMKII in BMMCs in response to $\mathrm{H}_{2} \mathrm{O}_{2}$; similar results were obtained in response to OVA (Figure 4D). These data suggest that an allergen can induce oxidative activation of CaMKII in mast cells and that this activation was prevented in MMVV $\delta$ mice.

To further determine whether ox-CaMKII can affect ROS production in mast cells, BMMCs were sensitized and challenged with OVA, following the same protocol in Figure 4C. Intracellular ROS production was measured at 3-minute intervals in these mast cells by using flow cytometry with CM-H2DCFDA, an indicator for general oxidative stress (Figure 4G). OVA-activated MMVV $\delta$ mast cells showed much lower levels of intracellular ROS compared with WT cells. Recent studies have suggested that mitochondrial ROS is an important source for promoting asthma phenotypes in OVA-challenged mice (38). We thus specifically detected mitochondrial ROS in these BMMCs using MitoTracker, a marker for mitochondrial localization, and MitoSOX, a 
A

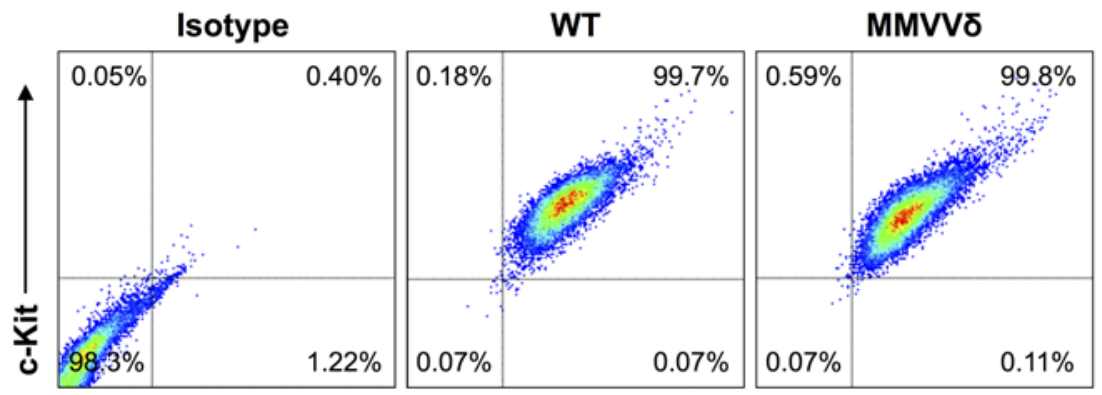

B

\section{FceRl-}
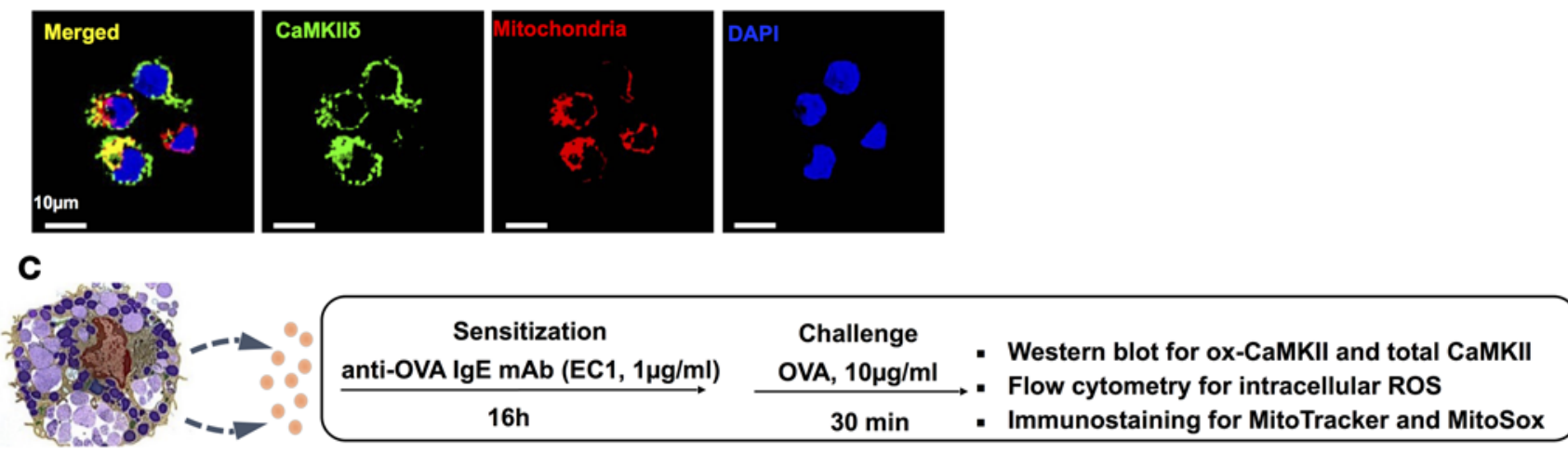

WT, MMVVס
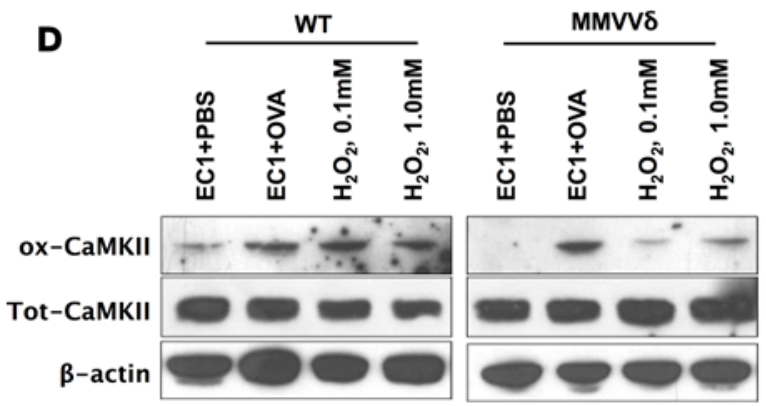

E
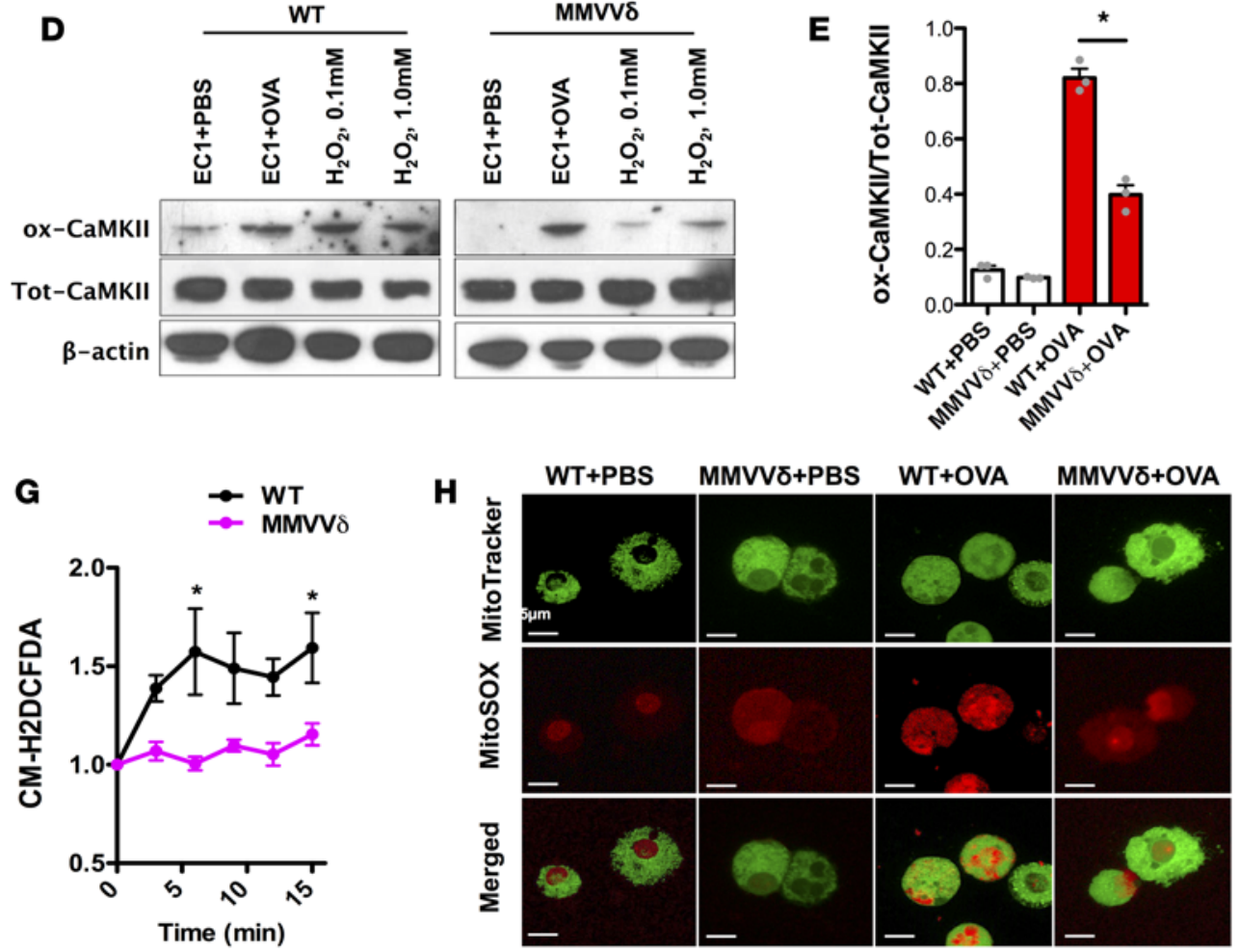
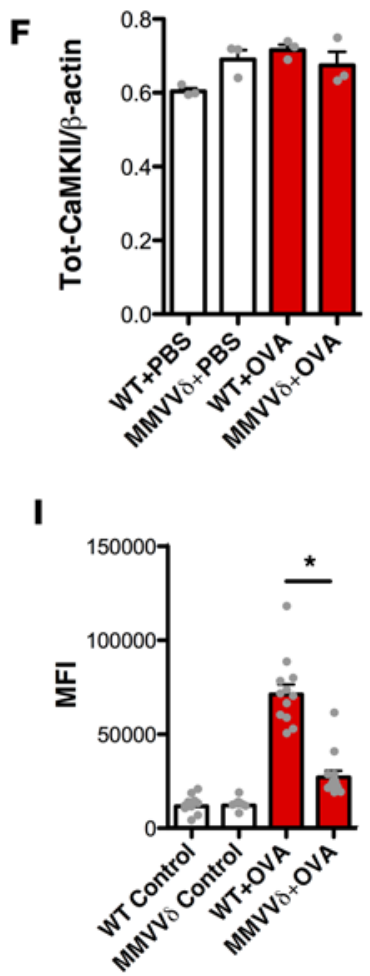

Figure 4. Reduced ROS levels in mast cells of ROS-resistant CaMKII mice. (A) Representation of BMMC FACS analysis for the surface markers c-Kit and FceRI. (B) Representation of images of the colocalization of CaMKII (green) and mitochondria (red) in BMMCs using confocal microscopy. Representative of 2 independent experiments. Scale bar: $10 \mu \mathrm{m}$. (C) Experimental setup for IgE-mediated mast cell activation and expression of CaMKII and ROS. BMMCs from WT and MMVV $\delta$ mice were sensitized with $1 \mu \mathrm{g} / \mathrm{ml}$ anti-OVA IgE (E-C1) for 16 hours and then stimulated with $10 \mu \mathrm{g} / \mathrm{ml} 0 \mathrm{VA}$ for 30 minutes. (D) Representative immunoblot of ox-CaMKII and total CaMKII (Tot-CaMKII) in OVA-sensitized and challenged BMMCs derived from WT and MMVV $\delta$ mice. Various dosages of $\mathrm{H} O$ were used as possible controls. One representative immunoblot of three is shown. (E and $\mathbf{F}$ ) Quantitative data for ox-CaMKII (E) and Tot-CaMKII (F) é2xpression using densitometry from 3 independent Western blots. Mean \pm SEM. (G) Levels of intracellular ROS in OVA-sensitized and challenged BMMCs by flow cytometry with CM-H2DCFDA. Mean \pm SEM, a single time point from 3 independent experiments. 
(H) Representative MitoTracker or MitoSOX staining of these OVA-sensitized and challenged WT and MMVV $\delta$ BMMCs from 3 independent experiments. Scale bar: $5 \mu \mathrm{m}$. (I) Quantitative data for MitoSOX expression in $\mathbf{H}$, as determined by mean fluorescent intensity (MFI). Data represent mean \pm SEM, 7-14 high power field view (HFV) per group. Comparisons were made using 2-tailed Student's $t$ test between OVA-treated WT vs. MMVV. ${ }^{*} P<$ 0.05 .

fluorescent mitochondrial ROS reporter (Figure $4 \mathrm{H}$ ). We found significantly increased levels of mitochondrial ROS in OVA-treated BMMCs compared with controls (Figure 4I). Similar to intracellular ROS, mitochondrial ROS levels were significantly reduced in MMVV $\delta$ BMMCs compared with WT cells. The same results were also found for the NADPH oxidase. Both p47 (Supplemental Figure 5A) and gp91phox (Supplemental Figure $5 \mathrm{~B}$ ) were markedly increased in OVA-activated BMMCs, but the increase was abolished in BMMCs from MMVV $\delta$ mice. These findings are consistent with our previous observations that ROS generation was elevated in OVA-activated mast cells (26) and, further, supported a view that ox-CaMKII $\delta$ may promote mitochondrial and NADPH oxidase-derived ROS production in allergen-activated mast cells.

ROS production is dependent on intracellular $\mathrm{Ca}^{2+}$. We have previously shown that the generation of intracellular ROS was dependent on increased intracellular calcium ([Ca $\left.{ }^{2+}\right]$ i) (26). To determine whether ROS production in MMVV $\delta$ mast cells is associated with changes in $\left[\mathrm{Ca}^{2+}\right]$, we first measured $\left[\mathrm{Ca}^{2+}\right] \mathrm{i}$ in OVA-activated WT and MMVV $\delta$ mast cells. While there was no detectable $\left[\mathrm{Ca}^{2+}\right] \mathrm{i}$ in mast cells in response to the calcium imaging buffer, OVA-activated mast cells exhibited elevated levels of $\left[\mathrm{Ca}^{2+}\right]$ (Figure 5A). Similar to the pattern of intracellular ROS production, MMVV $\delta$ mast cells showed significant reduction in $\left[\mathrm{Ca}^{2+}\right]$ i following OVA challenge compared with controls (Figure 5, B and C). We next tested the effect of an IP3 receptor antagonist, 2-aminoethoxydiphenyl borate (2-APB), on ROS production in these cells. Interestingly, we found that the OVA-activated ROS production was significantly blocked by 2-APB in WT cells (Figure 5D) and MMVV $\delta$ (Figure 5E) mast cells. These data suggest that the generation of intracellular ROS was dependent on elevated levels of $\left[\mathrm{Ca}^{2+}\right] \mathrm{i}$.

ox-CaMKII can modulate allergen-induced most cell activation. While ox-CaMKII was highly expressed in OVA-activated mast cells, up until this point the role of ox-CaMKII in regulating mast cell function remained elusive. To determine whether ox-CaMKII contributes to the activation of mast cells, mouse BMMCs from WT and ROS-resistant mast cells were sensitized and challenged with OVA, following the protocol in Figure 4C; mast cell degranulation ( $\beta$-hexosaminidase) and several mediators (e.g., histamine, LTC4, and cytokine IL-13) were detected and analyzed between mast cells from WT and MMVV $\delta$ mice. While PBS was unable to induce release of $\beta$-hexosaminidase, we detected enhanced degranulation in OVA-activated mast cells (Figure 6A). Moreover, compared with WT cells, MMVV $\delta$ mast cells showed a significant reduction in mast cell degranulation following OVA challenge. Similar results were obtained for histamine release (Figure 6B), LTC4 (Figure 6C), and IL-13 (Figure 6D). To further evaluate whether ox-CaMKII in mast cells has a functional effect on allergic diseases, we turned to an established model of IgE-mediated PCA, as illustrated in Figure 6E. Both WT and MMVV $\delta$ mice were passively sensitized with PBS or anti-OVA-specific IgE antibodies intradermally for 48 hours, and an immediate-type allergic reaction was induced by i.v. injection of OVA and Evans blue dye. An anaphylactic response was determined by the extravasation of Evans blue dye, indicative of vascular leakage (26). Compared with PBS, a significant enhancement of passive systemic anaphylaxis was noted in OVA-sensitized and challenged mice (Figure 6F). However, compared with WT mice, MMVV $\delta$ mice showed a reduced anaphylactic response. This suggests that ox-CaMKII determines allergen-induced mast cell signaling and anaphylaxis in vivo.

Adaptive transfer of WT mast cells reverses the protective effect of CaMKII MMVV in a mouse model of asthma. Given the significance of ox-CaMKII in modulating mast cell activation, we postulated that the ameliorated allergic airway inflammation in CRE- and HDM-treated MMVV $\delta$ mice may be due to ROS-resistant CaMKII expressed on mast cells. To test this hypothesis directly, CRE-challenged MMVV $\delta$ mice were administered BMMCs derived from MMVV $\delta$ or WT mice i.v. before CRE challenge (Figure 7A). We first assessed the efficiency of WT and MMVV $\delta$ mast cell recruitment in MMVV $\delta$ mice using our adoptive transfer model by analyzing the number of c-Kit ${ }^{+}$cells and ox-CaMKII in mast cells (i.e., costaining for both ox-CaMKII and c-Kit) in the lung tissues of these tested mice (Figure 7B). Specifically, we found that MMVV $\delta$ mice with adoptively transferred WT and MMVV $\delta$ BMMCs showed an increased number of mast cells (Figure 7C). Compared with untransferred BMMCs, the expression of ox-CaMKII was significantly increased in MMVV $\delta$ mice with adoptively transferred WT, but not MMVV8, BMMCs (Figure 7D). These findings indicate that these adoptively transferred BMMCs reached the lung and were activated by cockroach antigen challenge in our mouse model. We next investigated the effect of these adoptively transferred BMMCs on allergic inflammation in CRE-treat- 
A

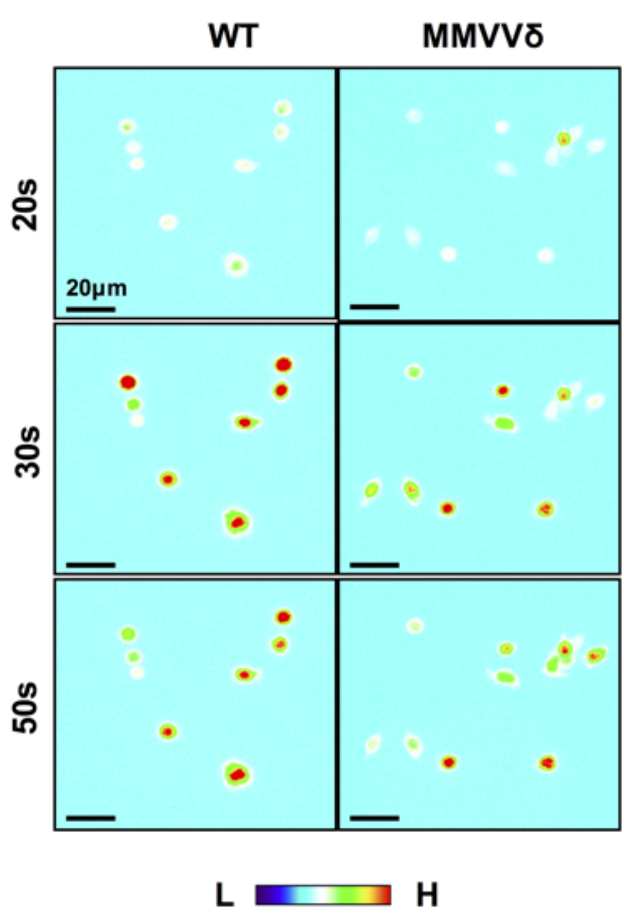

B

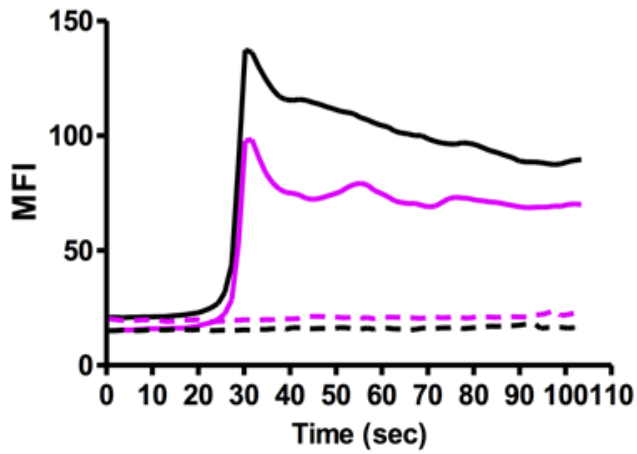

- - WT+CIB - WT+OVA

-- MMVV $\delta+C I B-M M V V+O V A$

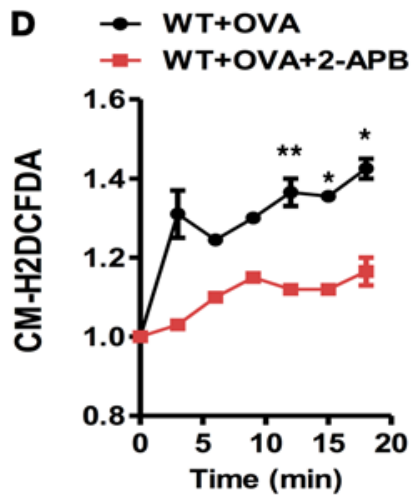

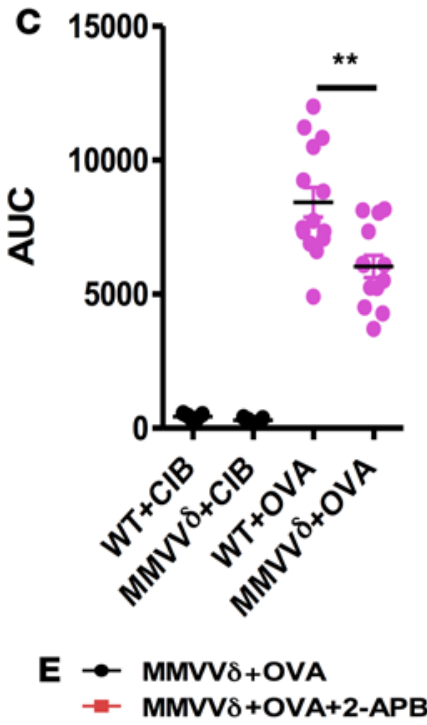

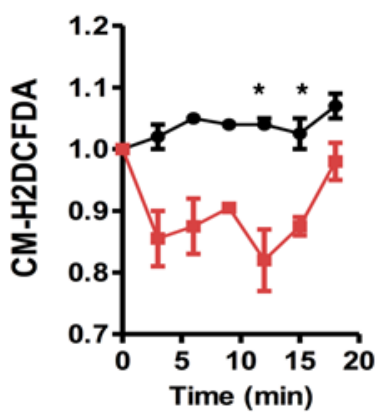

Figure 5. ROS production is dependent on intracellular $\mathrm{Ca}^{2+}$. (A) Representative Fluo-4 fluorescence heatmap images of anti-OVA IgE-sensitized BMMCs showing changes in [Ca $\left.{ }^{2+}\right]$ induced by OVA. Representative of 3 independent experiments. Scale bar: $20 \mu \mathrm{m}$. (B) Representative mean fluorescent intensity (MFI) average traces for sensitized and challenged BMMCs from WT and MMVV BMMCs. CIB, Ca ${ }^{2+}$ imaging buffer. (C) Quantification of total calcium response from sensitized and challenged cells ( $>150$ cells counted per condition) by calculating the AUC. (D and E) Levels of intracellular ROS in OVA-sensitized and challenged WT (D) or MMVV $\delta$ (E) BMMCs in the presence of IP3 receptor antagonist 2-APB by flow cytometry with CM-H2DCFDA. Data represent mean \pm SEM, a single time point from 3 independent experiments. Comparisons were made using 2-tailed Student's $t$ test between OVA-treated WT vs. MMVV group (C) or 2-APB-treated vs. nontreated OVA-activated WT (D) and MMVV $\delta$ BMMCs (E). ${ }^{*} P<0.05,{ }^{* *} P<0.01$.

ed MMVV $\delta$ mice. We found that the diminished peribronchial inflammation in MMVV $\delta$ mice was recovered by adaptive transfer of WT BMMCs but not by MMVV\& BMMCs (Figure 7E). Similar results were obtained for airway resistance (Figure 7F) and compliance (Figure 7G), BAL total cell counts (Figure 7H), eosinophils (Figure 7I), serum titers of CRE-specific IgE and IgG1 (Figure 7J), and Th2 cytokines IL-4, IL-5, and IL-13 in the BAL fluids (Figure 7K). Furthermore, the MMVV $\delta$ mice showed further increases in the levels of IFN- $\gamma$ in BAL fluid compared with WT mice (Supplemental Figure 6A). In contrast, when MMVV $\delta$ mice were adoptively transferred BMMCs from WT mice, increased levels of IFN- $\gamma$ were recovered, compared with MMVV $\delta$ mice with adoptive transfer of MMVV $\delta$ BMMCs. No changes were observed for IL-12 (Supplemental Figure 6B). These observations indicate that ox-CaMKII $\delta$ in mast cells may be critical in CRE-induced allergic inflammation and asthma in vivo.

A small-molecule CaMKII inhibitor suppresses OVA-induced mast cell activation, PCA, and asthma. A previous report found that KN-93, an experimental inhibitor of CaMKII (36), can suppress OVA-induced asthma (18). To further explore the possible role of CaMKII in mast cell activation and future treatment of asthma, we used KN-93 to treat OVA-activated BMMCs, following the protocol described in Figure 4A. We found a dose-dependent inhibition for IgE-mediated mast cell granulation (Figure 8A) and IL-13 secretion (Figure 8B). The findings were further validated in a human mast cell line (HMC-1) (Figure 8, C and D). Using the same experimental approach (Figure 6E), we found that KN-93 significantly inhibited OVA-induced PCA in vivo (Figure 8E). These findings, together with the previous report on the role of KN-93 in OVA-induced asthma (18), suggest that inhibition of CaMKII may prevent allergen-induced mast cell activation, allergic diseases, and asthma. 
A

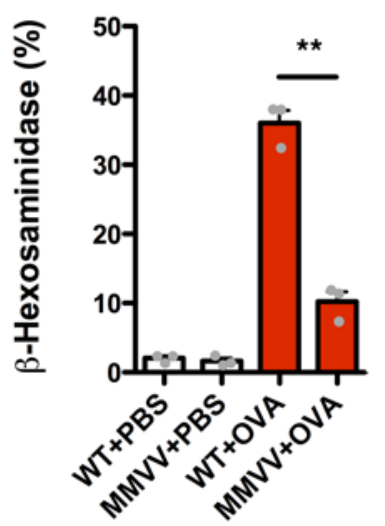

B

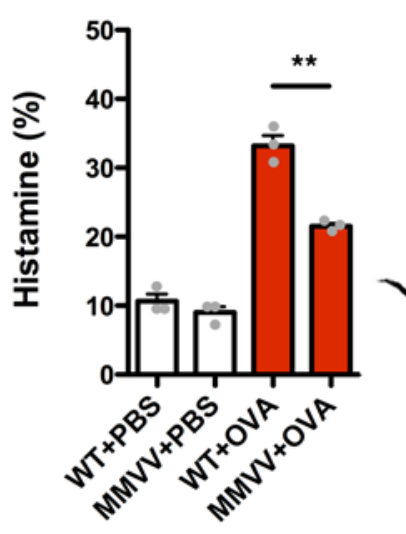

E

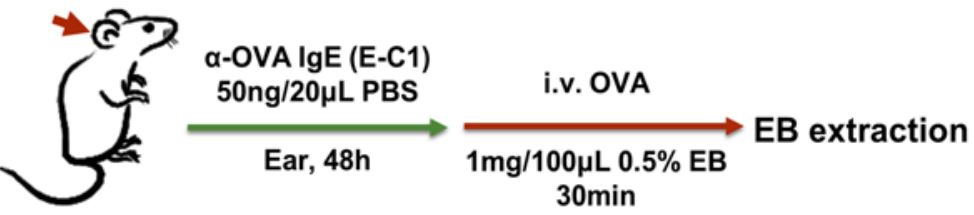

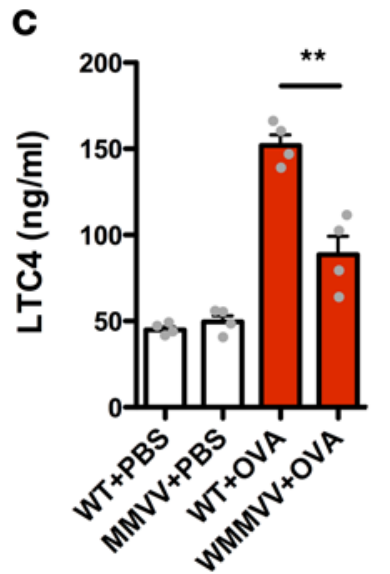

D

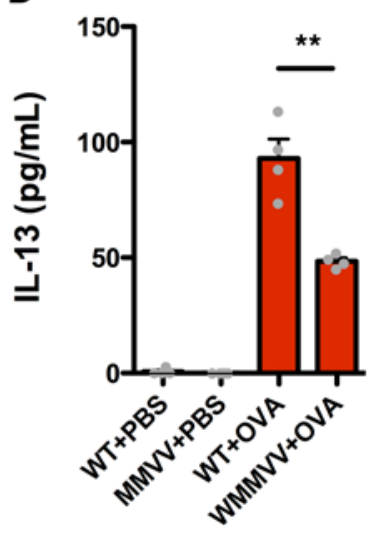

$\mathbf{F}$

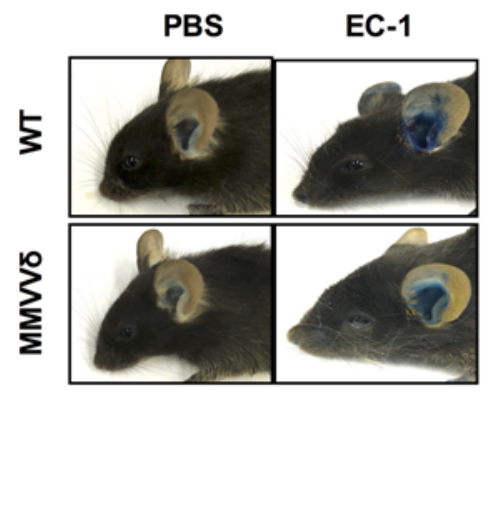

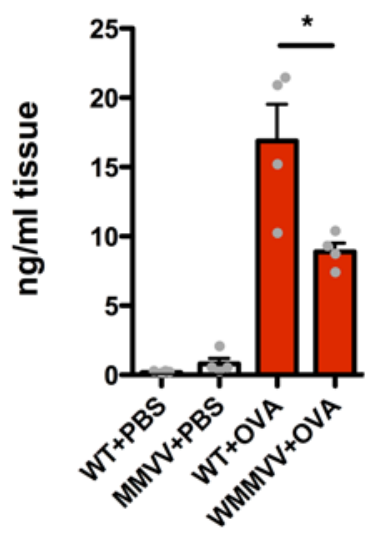

Figure 6. Oxidized CaMKII can modulate allergen-induced mast cell activation. (A-D) Levels of $\beta$-hexosaminidase (A), histamine (B), LTC4 (C), and IL-13 (D) in sensitized and challenged WT and MMVV $\delta$ BMMCs detected by ELISA. Mean \pm SEM, $n=4$ per group. (E) Experimental setup for IgE-mediated passive cutaneous anaphylaxis (PCA). Mice were injected intradermally with E-C1. After 48 hours, OVA was administered i.v. together with Evans blue dye for 30 minutes, followed by the quantification of the extravasation of Evans blue leakage into the skin. (F) Representative images of Evans blue-stained extravasation into skin 30 minutes after i.v. injection of OVA from WT and MMVV $\delta$ mice. For quantification of the extravasation of Evans blue leakage into the skin. Absorbance was measured at $620 \mathrm{~nm}$, and data are expressed as Evans blue in $\mathrm{ng} / \mathrm{mg}$ tissue. Data represent mean $\pm \mathrm{SEM}, n=4$ per group. Comparisons were made using 2-tailed Student's $t$ test between OVA-treated WT vs. MMVV group. ${ }^{*} P<0.05,{ }^{* *} P<0.01$.

\section{Discussion}

ox-CaMKII has recently been considered to be a critical mechanism linking ROS and downstream asthma phenotypes (9). Previous studies found that ox-CaMKII was significantly increased in the airway epithelium of asthmatic patients and correlated with asthma severity (18). Furthermore, by using human airway epithelial cells with ROS-resistant CaMKII MMVV overexpression, ox-CaMKII was found to be essential for increased $\mathrm{CI}^{-}$current, goblet cell metaplasia, and activation of NF-кB. In this study, we made an observation that exposure to environmental allergens can induce ROS generation and oxidative activation of CaMKII in mast cells. In addition, we suggested a possible mechanism that ox-CaMKII contributes to CRE-induced asthma through modulating mast cell activation. In particular, we have utilized MMVV $\delta$ mice by mutating methionines $281 / 282$ to valine in the isoform $\delta$. The MMVV mutant CaMKII $\delta$ is resistant to ROS activation without affecting $\mathrm{Ca}^{2+}$ - and calmodulin-dependent CaMKII activation and threonine 287 autophosphorylation (12). Thus, the MMVV $\delta$ mouse is uniquely suited to directly examine whether CaMKII oxidation at methionines $281 / 282$ is critical in response to excessive ROS and the pathogenesis of allergen-induced asthma and allergic diseases.

First, we used the MMVV $\delta$ mouse to generate a CRE-induced mouse model of asthma to investigate the significance of the ROS-resistant MMVV $\delta$ in allergen-induced asthma. Indeed, CRE-challenged MMV$\mathrm{V} \delta$ mice showed a significant reduction of several typical asthma-related features, including airway inflam- 
A

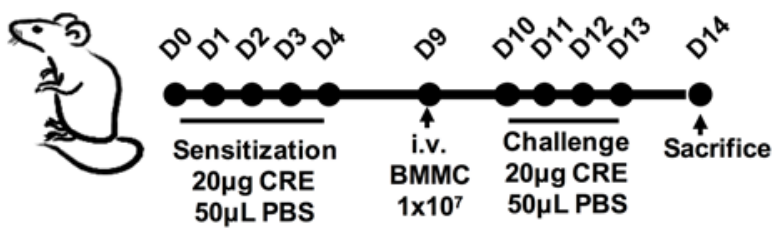

C

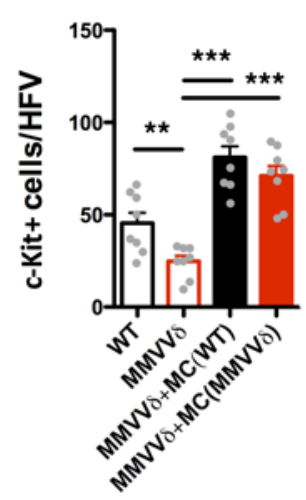

E

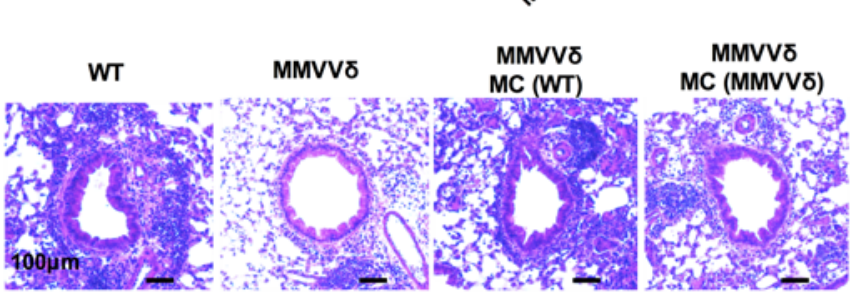

D
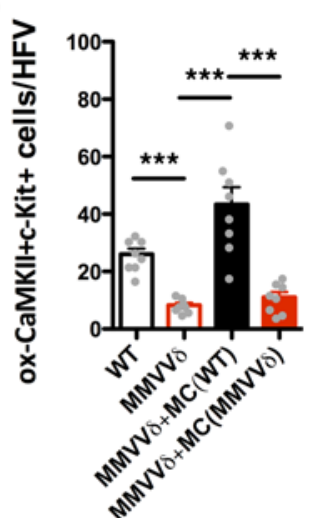

B

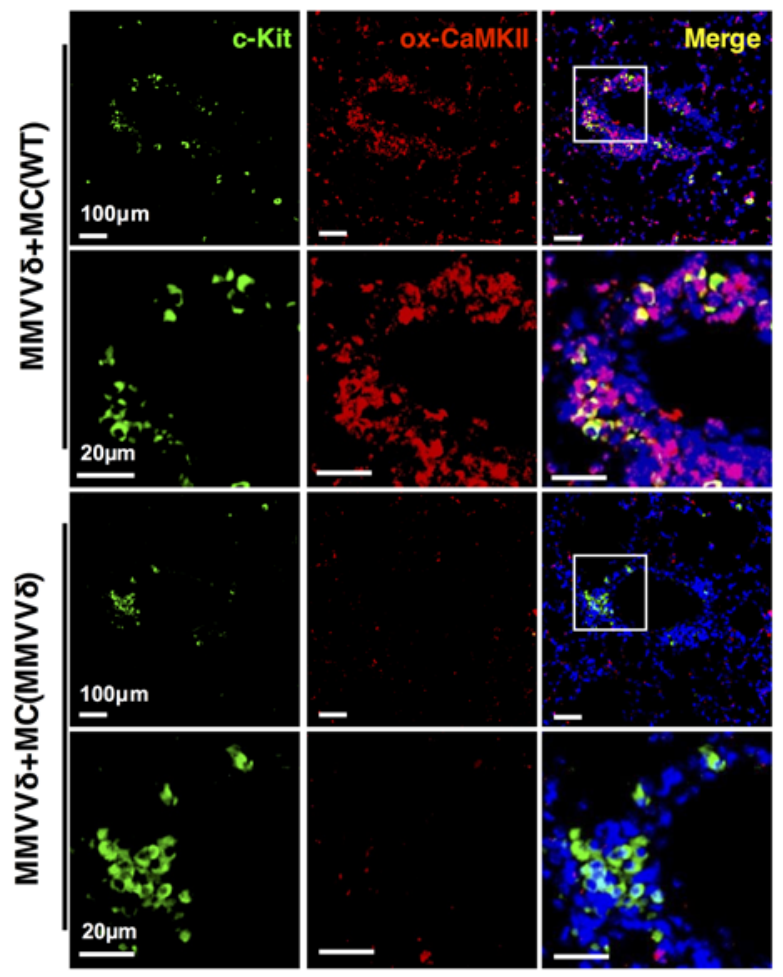

$\mathbf{F}$

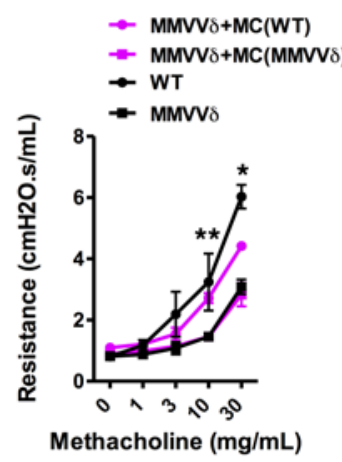

H
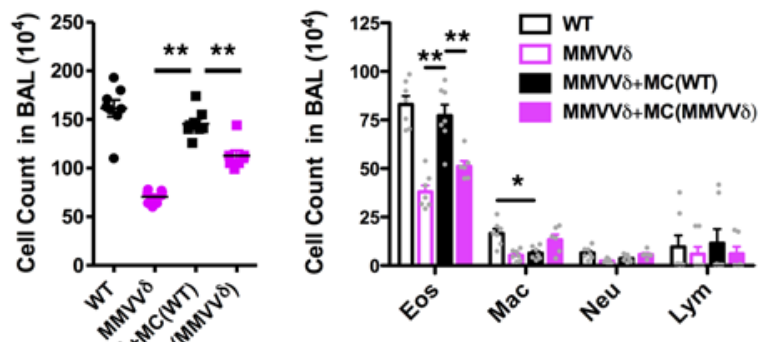

I
$\mathbf{J}$

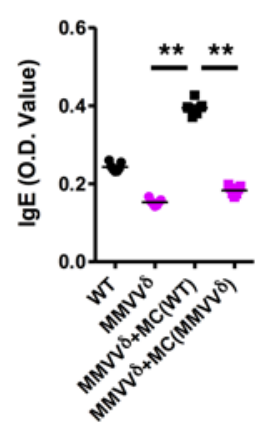

G

$\rightarrow-M M V V \delta+M C(W T)$

- MMVVס $+M C(M M V V \delta)$

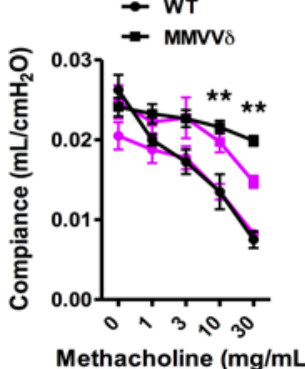

and

$\mathbf{K}$

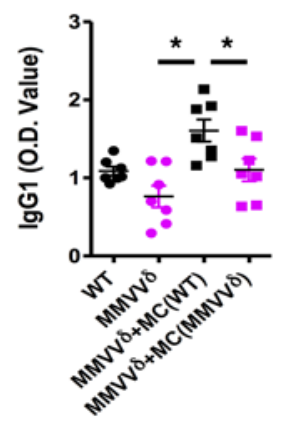

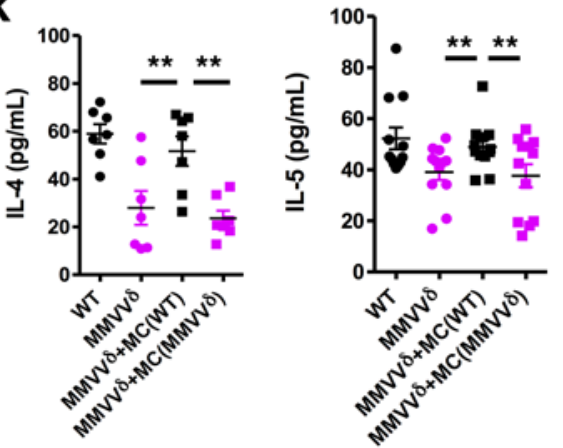

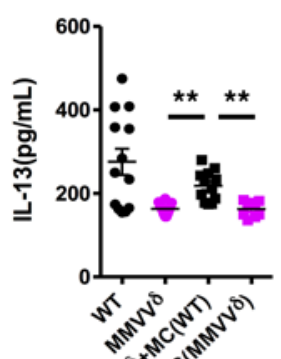


Figure 7. Adoptive transfer of WT mast cells reverses the protective effect of CaMKII MMVV $\delta$ in a mouse model of asthma. (A) Protocol for adaptive transfer of BMMCs in the cockroach allergen-induced mouse model of asthma. (B) Colocalization of c-Kit (green) and ox-CaMKII (red) in the lung sections of MMVV $\delta$ mice with adoptively transferred WT and MMVV $\delta$ BMMCs. Scale bar: $100 \mu \mathrm{m}$ (first and third row); $20 \mu \mathrm{m}$ (second and fourth row). (C and D) Numbers of c-Kit-positive cells (C) and cells positive for both c-Kit and ox-CaMKII (D) from WT and MMVV $\delta$ mice with adoptive transfer of WT and MMVV $\delta$ BMMCs. Mean \pm SEM, $n=8$ high power field view (HFV) per group. (E) Representation of H\&E-stained paraffin lung tissue sections from WT and MMVV $\delta$ mice adoptively transferred with WT or MMVV $\delta$ BMMCs in a CRE-induced mouse model of asthma (6 mice per group). Scale bar: $100 \mu \mathrm{m}$. (F and $\mathbf{G})$ Systemic airway resistance $(\mathbf{F})$ and compliance $(\mathbf{C})$ in response to increasing concentrations of methacholine using the forced oscillation technique (FlexiVent, SCIREQ). Data represent mean \pm SEM, 6 mice per group. $(\mathbf{H}$ and $\mathbf{I})$ Bronchoalveolar lavage $(B A L)$ total $(\mathbf{H})$ and differential (I) cell counts of CRE-challenged WT and MMVV $\delta$ mice. Mean \pm SEM, 7 mice per group. (J) Serum levels of cockroach allergen-specific lgE and IgG1. Mean \pm SEM, 7 mice per group. (K) Levels of IL-4 $(n=7)$, IL-5 $(n=12)$, and IL-13 $(n=12)$ in BALs. Data represent mean \pm SEM; comparisons were made using 2-tailed Student's $t$ test of CRE-treated MMVV $\delta$ vs. MMVV $\delta$ with adoptively transferred WT BMMCs and MMVV $\delta$ with adoptively transferred WT vs. MMVV $\delta$ BMMCs. ${ }^{*} P<0.05,{ }^{* *} P<0.01,{ }^{* *} P<0.001$.

mation, goblet cell hyperplasia, AHR, and recruitment of eosinophils in the BAL fluids. Additionally, these mice showed a significant reduction in CRE-specific IgE and IgG1 and Th2 responses. These data provided supportive evidence for a role of ox-CaMKII in CRE-induced airway inflammation and allergic asthma. Furthermore, these phenotypic changes in CRE-induced asthma were validated when the mice were exposed to another common allergen, HDM. Compared with the CRE, a comparatively modest effect of MMVV $\delta$ knockin on airway resistance was noted, which may be due to the experimental settings that were optimized for CRE. Namely, the peak for airway resistance in the HDM model might have not yet been achieved, as it would for CRE. Importantly, our data suggest that MMVV $\delta$ protects against allergen-induced AHR and lung inflammation in either CRE- or HDM-induced mouse models of asthma. These allergen-induced mouse models of asthma, particularly the well-established CRE-induced mouse model of asthma in our laboratory $(33,37)$, are relevant to patients with asthma and mimic those asthmatic patients who are allergic to CRE. CRE exposure and sensitization has been identified as one of the strongest risk factors associated with the development, severity, and morbidity of asthma (39-41). CREs are detected in $85 \%$ of inner city US homes and $60 \%-80 \%$ of inner city children with asthma are sensitized to CRE (42).

We next examined levels of ROS in a CRE-induced mouse model of asthma and the effect of ROS-resistant MMVV $\delta$ on ROS production. ROS plays a significant role in pollen-induced (7) or HDM-induced (8) allergic sensitization and asthma. Very recent studies have suggested that HDM can directly induce ROS generation, causing oxidative DNA damage and asthma-associated pathophysiology $(8,43)$. Environmental allergens can either directly induce ROS production or promote local inflammatory processes that lead to the secondary production of ROS (6-8). Here, we demonstrated that CRE can induce ROS production and that the production was suppressed in ROS-resistant MMVV $\delta$ mice, suggesting that ox-CaMKII may participate in a feed forward circuit to increase ROS production in mast cells. Although the molecular details of such a circuit remain to be determined, we observed an increased expression of ox-CaMKII in CRE-challenged WT mice that was significantly decreased in ROS-resistant MMVV\& mice. We interpret this finding to suggest that oxidative activation of CaMKII may be related to ROS generation by transcriptional and posttranscriptional mechanisms. It is known that various factors and their signaling pathways, such as cytokines and endotoxin (44), hyperglycemia (15), angiotensin (12, 17, 45), aldosterone (14), and intracellular $\mathrm{Na}^{+}$overload (46), contribute to the generation of excessive ROS, leading to increased ox-CaMKII. However, the potential mechanisms and upstream pathways that are associated with the increased allergen-induced ROS generation remain unknown. Previous studies have suggested that myocardial ox-CaMKII expression may be at least partially dependent on ROS generated by activation of the TLR4 and myeloid differentiation factor 88 (MyD88) pathway (44). Thus, it is possible that CRE used for the experiment may contain sufficient TLR4 ligand to activate TLR4 signaling and contribute to ROS generation, airway reactivity, and inflammation. Additionally, TLR7 (47) and proteinase-activated receptor-2 (PAR-2) (48) have been associated with allergen-induced ROS generation. $\mathrm{AhR}$, a receptor for environmental pollutants, plays a crucial role in ROS production (49) and immune regulation $(50,51)$. Our studies demonstrated that $\mathrm{AhR}$ is important in allergen-induced ROS generation and mast cell activation (26). Taken together, these findings suggest that multiple signaling pathways may contribute to the generation of ROS that leads to the oxidative activation of CaMKII. It is likely that CaMKII acts as a key signaling node for convergence of multiple upstream activators that operate by increasing ROS, while ox-CaMKII, in turn, enhances ROS generation by actions at downstream targets. Although the intracellular source of the ROS is unlikely to be delineated, both NADPH oxidase $(12,14)$ and mitochondria $(15,16)$ are considered as major sources of ROS that are induced by external stimuli 
A

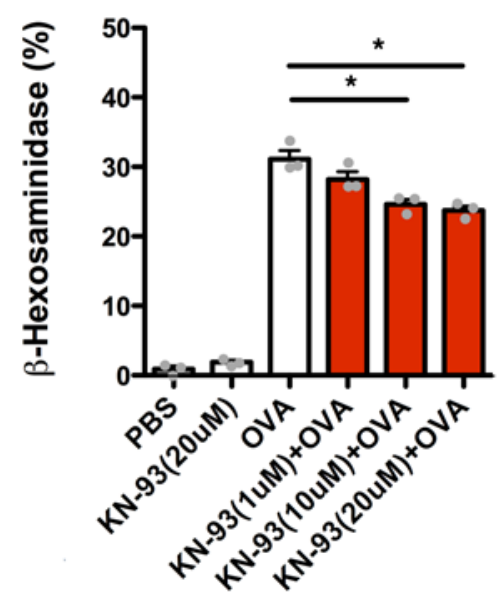

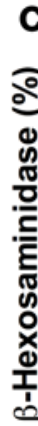

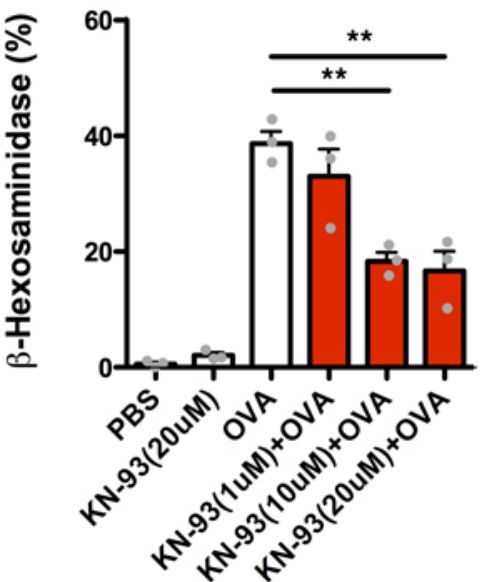

B

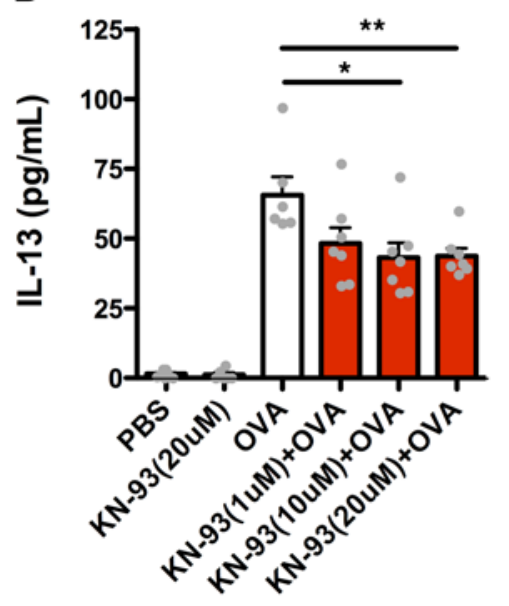

D

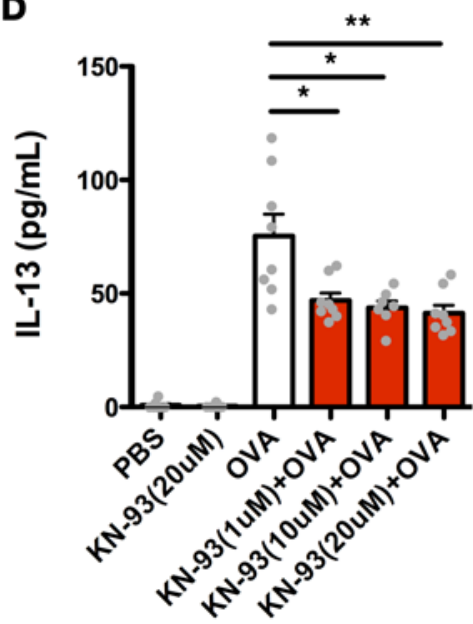

$\mathbf{E}$
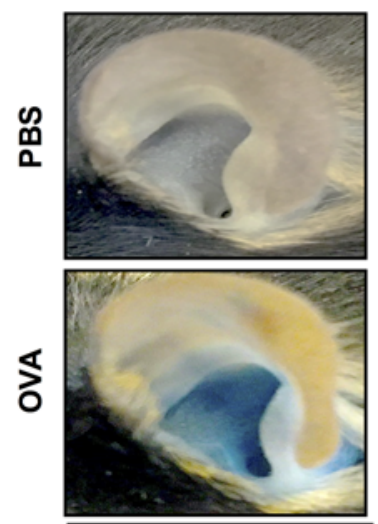

हू

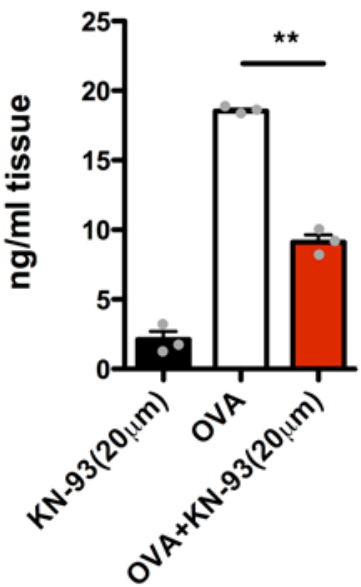

Figure 8. CaMKII inhibitor suppresses OVA-induced mast cell activation and PCA in vivo. BMMCs or human mast cells, HMC-1 cells, were sensitized with $1 \mu \mathrm{g} / \mathrm{ml}$ anti-OVA IgE (E-C1) in the presence or absence of different doses of KN-93 (1-20 $\mu \mathrm{M})$ for 16 hours and then stimulated with PBS or $10 \mu \mathrm{g} / \mathrm{ml}$ OVA for 30 minutes. (A-D) Mast cell activation was assessed by measuring $\beta$-hexosaminidase $(n=3)$ in BMMCs (A) and HMC-1 cells (C) and IL-13 levels ( $n=6)$ in BMMCs (B) and HMC-1 cells (D). (E) Representative images of Evans blue-stained extravasation into skin. Mice were injected intradermally with E-C1 with or without KN-93. After 24 hours, OVA was administered i.v. together with Evans blue dye for 30 minutes, followed by the quantification of the extravasation of Evans blue leakage into the skin. Data represent mean \pm SEM, 3 mice per group. Comparisons were made using 2-tailed Student's $t$ test between OVA-treated mast cells vs. mast cells pretreated with different dosages of $\mathrm{KN}-93$ and then challenged with OVA (A-D) or mice pretreated with or without $\mathrm{KN}-93$ and then challenged with OVA $(\mathrm{E}) .{ }^{*} P<0.05,{ }^{* *} P<0.01$.

(52). This was supported by our studies indicating that both mitochondrial ROS and NADPH oxidase (gp91phox and p47) were markedly increased in OVA-activated BMMCs but were abolished in BMMCs from MMVV $\delta$ mice, suggesting that MMVV $\delta$ may affect mitochondrial ROS generation, the activation of NADPH oxidase, and subsequently, the oxidative activation of CaMKII.

Previous studies have focused on CaMKII activity and function in airway epithelial and airway smooth muscle cells. Differential expression of ox-CaMKII in human airway epithelium but not in smooth muscle cells was observed in samples obtained from asthmatic patients and healthy controls (18), suggesting that ox-CaMKII promoted asthma through actions in airway epithelium. Our results substantially expand this initial observation by showing that mast cell activation by ox-CaMKII contributes to key asthma phenotypes.

One of the major events in allergic inflammation is the increase in mast cells at the site of tissue mucosa where they can respond to foreign organisms, environmental pollutants, and antigens $(35,53)$. Considering the critical role of mast cells in allergic diseases and their strategic location, we specifically focused on mast cells and examined the role of ox-CaMKII on mast cells in modulating mast cell function and subsequent contribution to the development of asthma. Not surprisingly, mast cell numbers were significantly increased in the lung tissues of CRE-treated mice. However, compared with WT mice, MMVV $\delta$ mice showed fewer 
mast cells, suggesting that ox-CaMKII may also affect mast cell recruitment to the inflammatory sites following allergen challenge. While many factors may contribute to the mast cell migration (e.g., RANTES; CCL2; MCP-1; CXCR4, ref. 54, 55; and CXCR8, ref. 56), tryptase has recently been considered one of the major factors released from mast cells contributing to cell accumulation (53). Indeed, we found that MMVV $\delta$ BMMCs released less tryptase compared with WT cells upon challenge (data not shown). Thus, it is likely that ox-CaMKII may promote tryptase release from activated mast cells, leading to the recruitment of mast cells in asthma. Indeed, ox-CaMKII expression was increased in mast cells of lung tissues of CRE-challenged WT mice but reduced in those of MMVV $\delta$ mice. These findings were further confirmed in isolated and cultured BMMCs, following our well-established protocol for IgE-mediated activation of mast cells (26), suggesting that ox-CaMKII may modulate ROS generation in mast cells. However, the exact mechanism remains unclear. Our previous studies suggested that the increased $\left[\mathrm{Ca}^{2+}\right]$ i may contribute to the generation of ROS (26). Indeed, MMVV $\delta$ mast cells with reduced ROS generation showed a significant reduction in $\left[\mathrm{Ca}^{2+}\right]$ compared with control mast cells. Furthermore, ROS generation was significantly inhibited by calcium antagonist in WT and MMVV $\delta$ mast cells. These findings suggest that the actions of ox-CaMKII to increase ROS occur, at least in part, by a $\mathrm{Ca}^{2+}$-dependent mechanism. It would be of interest and importance to learn how ox-CaMKII affects $\mathrm{Ca}^{2+}$ signaling, particularly the influx of exogenous calcium.

One of the most significant observations we made in this study is that ox-CaMKII participates in modulating mast cell activation. This is consistent with our previous studies, indicating that the generation of $\left[\mathrm{Ca}^{2+}\right]$ i and ROS contributes to mast cell activation, including degranulation and LCT4 production (26). Thus, we postulated that ox-CaMKII regulates $\left[\mathrm{Ca}^{2+}\right] \mathrm{i}$ and ROS production and subsequently leads to the activation of mast cells that define allergic asthma. Indeed, OVA-challenged MMVV $\delta$ mast cells showed significantly reduced activation, as assessed by degranulation, histamine release, LTC4, and IL-13 compared with control cells. Furthermore, ox-CaMKII contributes to IgE-mediated anaphylactic response. Furthermore, a significant enhancement of passive systemic anaphylaxis was noted in OVA-sensitized and challenged control mice but not in MMVV $\delta$ mice. The positive actions of ox-CaMKII on mast cell activation were further supported by the investigation of IgE-initiated signaling events that are associated with mobilization of $\left[\mathrm{Ca}^{2+}\right] \mathrm{i}$, degranulation, and release of inflammatory and allergic mediators in mast cells (Syk, PLC $\gamma 1$, p38, ERK, and Akt) (data not shown). Taken together, these data provided evidence that ox-CaMKII may amplify antigen-challenged mast cell activation. In addition, we recognize that our studies focused on the IgE receptor FceRI-dependent pathway in mast cells (23-25) and that ox-CaMKII regulation of non-IgE-mediated mast cell activation, such as TLRs $(57,58)$ or Mrgprb2/MRGPRX2-mediated (a gene uniquely expressed on mast cell) (59) mast cell activation, remains unexplored.

Given the apparent significance of ox-CaMKII in modulating mast cell activation in vitro, we tested whether ox-CaMKII-activated mast cells may promote allergen-induced allergic inflammation and asthma. We have assessed the efficiency of mast cell recruitment to the MMVV $\delta$ mice in the adoptive transfer experiment by analyzing the number of $\mathrm{c}-\mathrm{Kit}^{+}$cells and ox-CaMKII in the lung mast cells of these tested mice. Indeed, these MMVV $\delta$ mice with adoptively transferred BMMCs showed increased number of mast cells, and the lower ox-CaMKII in MMVV $\delta$ mice was markedly recovered by adoptively transferred WT, but not MMVV\&, BMMCs. These findings indicate that these adoptively transferred BMMCs reached lung and were activated by cockroach antigen challenge in our mouse model. In fact, we have tracked the migration of i.v.-injected $\mathrm{GFP}^{+}$bone marrow-derived mesenchymal stem cells (MSCs) and found a significant amount of the migrated $\mathrm{GFP}^{+} \mathrm{MSCs}$ in the lung tissues after CRE challenge (33). As expected, these MMVV $\delta$ mice with adoptively transferred WT BMMCs, but not MMVV $\delta$, reversed the ameliorated AHR and allergic airway inflammation in MMVV $\delta$ mice, suggesting that ox-CaMKII $\delta$ in mast cells is crucial for the CRE-induced allergic inflammation and asthma. Given the significance of ox-CaMKII in mast cells, it would be of interest to see the role of mast cells with or without ox-CaMKII in allergen-induced asthma by developing mast cell-specific oxidant-resistant CaMKII MMVV knockin mice. However, no ideal mast cell-specific Cre mice are available, and such an endeavor is beyond the scope of this initial study. Additionally, we showed that a small-molecule CaMKII inhibitor, KN-93, inhibited IgE-mediated mast cell granulation and IL-13 secretion in both human and mouse mast cells and anaphylactic response in vivo. These findings further suggest that CaMKII inhibition may be a new and effective antioxidant therapy for asthma and allergic diseases.

Taken together, previous works have demonstrated that NADPH oxidases are required for oxidative activation of CaMKII by the use of p47 knockout mice (18). Moreover, CaMKII oxidation can be reversed by MsrA (60), and $\mathrm{MsrA}^{-/-}$OVA-challenged mice showed exaggerated CaMKII oxidation and exhibited a 
increase in goblet cell hyperplasia, bronchial thickness, and eosinophils. Furthermore, studies on mice with epithelial-targeted transgenic expression of a CaMKII inhibitory peptide suggest that bronchial epithelial ox-CaMKII was required to increase a ROS- and picrotoxin-sensitive $\mathrm{Cl}^{-}$current and MUC5AC expression, upstream events in asthma progression (18). In this study, we have focused on the role of ox-CaMKII in the pathogenesis of asthma by using ROS-resistant CaMKII MMVV $\delta$ mice. We provided evidence that ox-CaMKII contributes to the pathogenesis of asthma by modulating mast cell activation. These studies link the CRE-ROS-ox-CaMKII axis to mast cell activation and subsequent development of asthma and allergic diseases. Our findings suggest that the development of potent and specific inhibitors of CaMKII could offer a novel approach to the treatment of asthma.

\section{Methods}

Additonal details can be found in the Supplemental Methods

Animals. Six- to eight-week-old mice were used in the experiment. C57BL/6 WT mice were purchased

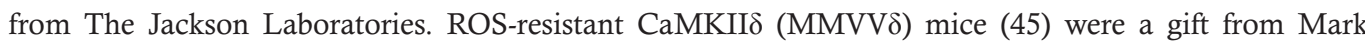
Anderson's laboratory at Johns Hopkins University (Baltimore, Maryland, USA). Age-matched mice were used as controls. All mice used in this study are from the B6 background. All of the mice were maintained under specific pathogen-free conditions in our animal facility on a 12-hour light/12-hour-dark cycle, with free access to food and water.

Allergen-induced asthma mouse model. The CRE-induced asthma mouse model was established as described previously $(33,37)$. Briefly, mice were sensitized by intratracheal inhalation of $20 \mu \mathrm{g}$ cockroach extract (CRE, B46, GREER Laboratories) in $50 \mu \mathrm{l}$ of PBS under light anesthesia with isoflurane on days $0-4$. Mice were subsequently challenged on days 10-13 with the same amount of CRE. Control mice received saline during the sensitization and challenge phases. On day 14, mice were tested for lung function and then sacrificed, BAL fluid was harvested for total and differential counts of lavage cells and ELISA measurements, and lung tissues were dissected for histological analyses. Blood was taken to screen for serum antibodies against CREs. The same protocol was applied to HDM. For KN-93 treatment, intratracheal administration of $20 \mu \mathrm{l}$ of $100 \mu \mathrm{M} \mathrm{KN}-93$ or deionized $\mathrm{H}_{2} \mathrm{O}$ was performed 30 minutes before CRE challenge on days 10-13, following the same protocol.

Analysis of lung inflammation. For analysis of BAL fluids, lavage fluids were centrifuged at $300 \mathrm{xg}$ for 10 minutes at $4^{\circ} \mathrm{C}$ and washed. Red blood cells in the pellet were lysed by ammonium-chloride-potassium lysis buffer. Cellular differential percentages were determined by means of flow cytometry on a FACS Calibur cytometer (BD Biosystems), and the data collected were analyzed with Flowjo software (Treestar) (37). Eosinophils were defined as SSC ${ }^{\text {hi }}$ SiglecF $^{+}$(clone E50-2440, BD) Mac-3- (M3/84, BD) cells, alveolar macrophages cells were identified as $\mathrm{SSC}^{\text {hi }}$ SiglecF $\mathrm{Fac}^{+} 3^{+}$cells, granulocytes were recognized as SSC ${ }^{\text {hi }} \mathrm{Gr}-1^{+}$ (clone RB6-8C5, eBioscience) cells, and lymphocytes were identified as $\mathrm{FSC}^{\mathrm{lo}} / \mathrm{SSC}^{\mathrm{lo}}$ and expressing CD3 (clone 145-2C11, eBioscience) or CD19 (clone 1D3, eBioscience). For the histological assessment of lung inflammation, mouse lungs were perfused with $10 \mathrm{ml}$ ice-cold PBS injected into right ventricle followed by excision and fixation in $4 \%$ neutral buffered formalin. Sections $(5 \mu \mathrm{M})$ were prepared from paraffin-embedded lung tissues and stained with $\mathrm{H} \& \mathrm{E}$ and periodic acid-Schiff (PAS). Images were obtained using a NIKON ECLIPSE Ti-U microscope equipped with DS-Fi2 camera (NIKON, USA).

Lung function. Mice were anesthetized with a ketamine $(90 \mathrm{mg} / \mathrm{kg}) / x y l a z i n e ~(18 \mathrm{mg} / \mathrm{kg})$ mixture, and a tracheotomy tube was inserted. Ventilation was initiated with a volume-cycled ventilator (Flexivent; SCIREQ Scientific) with a positive-end expiratory pressure of $\left.2 \mathrm{cmH}_{2} \mathrm{O}\right)$. Airway responsiveness was measured by challenging mice to increasing doses of aerosolized methacholine $(0-30 \mathrm{mg} / \mathrm{ml})$. The pulmonary resistance and compliance were measured with the Flexivent software and exported to Pulmodyn data-acquisition software (Hugo Sachs Electronic) for further data analysis.

ELISA. Cytokines (e.g., IL-4, IL-5, and IL-13) in the BAL fluids of mice or culture supernatants of mast cells were measured by ELISA using the Ready-Set-Go! ELISA sets (eBioscience). LTC4 in supernatants of mast cells was measured by ELISA according to the manufacturer's instructions (Cayman Chemical). Serum CRE-specific IgE and IgG1 were also analyzed by ELISA as described previously (37).

Immunofluorescence and immunostaining. Lung sections were blocked with protein-blocking serumfree solution (Dako) and incubated with the primary antibodies overnight at $4^{\circ} \mathrm{C}$. After the sections were washed with TBST and incubated with fluorescent dye-conjugated secondary antibodies at room temperature for 1 hour, nuclear staining was carried out with 6-diamidino-2-phenylindole, dihydrochloride (DAPI) 
(ThermoFisher). The following antibodies were used: ox-CaMKII (catalog 01-1387, Millipore), CaMKII (catalog 15443-1-AP, Proteintech), tryptase (clone AA1, Abcam), c-Kit (clone AK2, eBioscience), Mitotracker Green FM (ThermoFisher), and MitoSOX Red (ThermoFisher). The detection of intracellular superoxide in lung tissues was carried out using dihydroethidium (DHE) (ThermoFisher). To determine the fluorescence signal in tissue sections, fluorescent cells in 5 different high-power fields from each slide were quantified using ImageJ v1.50e (NIH) and presented as mean fluorescence intensity per square micrometer. Two to three slides from each sample were used for analysis.

Confocal microscopy. Images were captured by Cell Observer Z1 (Carl Zeiss) with a LSM780 scanner (Carl Zeiss) using a $\times 40$, NA 0.95 Corr Plan-Apochromat objective (Carl Zeiss) and then were analyzed using Zen Black, Zen Blue, or AxioVision 4.2 software (Carl Zeiss). Fluorescence signals of CaMKII $\delta$, mitochondria, and nucleus were visualized by fluorescence with excitation at 488, 561, and $405 \mathrm{~nm}$, respectively.

$D A B$ staining. After antigen retrieval of deparaffinized embedded tissue sections, the slides were blocked with $3 \%$ BSA containing $0.1 \%$ TX100 and $0.1 \% \mathrm{NaN}_{3}$ for 1 to 2 hours at $37^{\circ} \mathrm{C}$ with rocking. The primary antibody solution was made at 1:200 dilution with anti-mast cell tryptase antibody (clone AA1, Abcam) overnight at $4^{\circ} \mathrm{C}$. The slides were washed with TBST, followed by incubation with anti-mouse IgG-HRP (catalog SC-2005, Santa Cruz) for 2 hours at $37^{\circ} \mathrm{C}$, and then developed by using DAB detection system (DAKO).

Western blotting. 30 - to $50-\mu \mathrm{g}$ protein aliquots were boiled and loaded onto $12 \%$ Tris-Tricine Gels (Invitrogen) electrophoresed in running buffer (NuPAGE MES SDS Running Buffer; Invitrogen) and then transferred to polyvinylidene difluoride membranes (Invitrogen). Membranes were incubated with the primary antibody for overnight at $4^{\circ} \mathrm{C}$ and then probed with the HRP-conjugated secondary antibody (goat anti-rabbit or mouse IgG-HRP, catalog SC-2005, Santa Cruz, 1:2,000) for 1 hour at $37^{\circ} \mathrm{C}$. Detection was performed by the ECL Western blotting detection system (GE Life Sciences). Primary antibodies include ox-CaMKII (catalog 01-1387, Millipore), total CaMKII (clone EP1829Y, Abcam), and $\beta$-actin (clone 4E8H3). All these were diluted to 1:1,000. The blots were imaged by a Luminescent Image Analyzer LAS-4000 Mini (Fujifilm) and quantified by MultiGauge version 3. 2 program (Fujifilm).

$B M M C$ culturing. BMMCs were obtained by culturing mouse bone marrow cells as described previously (26). Briefly, bone marrow cells were cultured at a starting density of $5 \times 10^{5} \mathrm{cells} / \mathrm{ml}$ in the presence of $30 \%$ WEHI-3-conditioned medium as a source of IL-3 for the first 3 weeks, which was then changed to $10 \mu \mathrm{g} / \mathrm{ml}$ IL-3 for an additional 1 to 3 weeks. Mast cell phenotype was confirmed by flow cytometry analysis with Abs specific for c-Kit (eBiosciences) and FceRI (eBiosciences) or by histochemical staining with acid toluidine blue.

Measurements of degranulation and histamine release. Harvested BMMCs were sensitized with $1 \mu \mathrm{g} / \mathrm{ml}$ antiOVA IgE (clone E-C1, Chondrex Inc.) and incubated overnight at $37^{\circ} \mathrm{C}$ in a microplate in a humidified atmosphere containing $5 \% \mathrm{CO}_{2}$. They were then challenged with $10 \mu \mathrm{g} / \mathrm{ml}$ OVA in Tyrode's buffer for 30 minutes at $37^{\circ} \mathrm{C}$. Degranulation was monitored by measuring $\beta$-hexosaminidase release in the culture supernatants. Unstimulated cell lysate prepared with Tyrode's buffer containing $0.5 \%$ TritonX-100 was used to obtain the total $\beta$-hexosaminidase content. Degranulation is expressed as a percentage of total $\beta$-hexosaminidase activity in the input cells. Histamine release was assessed by using automated fluorimetry and reported as a percentage of total histamine content determined by lysing an equivalent number of mast cells (61).

Passive cutaneous anaphylaxis. Mice were lightly anesthetized with ketamine and xylazine and sensitized by intradermal injection of $50 \mathrm{ng}$ anti-OVA IgE diluted in $20 \mu \mathrm{PBS}$ in the ear; vehicle control (PBS) was given in the contralateral side of ear as a control. After 48 hours, the mice were injected i.v. with $1 \mathrm{mg}$ OVA in $100 \mu 10.5 \%$ Evans blue dye in saline for 30 minutes, and the mice were then sacrificed by terminal anesthesia. Tissue sections around the intradermal injection sites were excised, and blue dye was extracted with formamide at $55^{\circ} \mathrm{C}$ overnight. Absorbance was measured at $620 \mathrm{~nm}$, and data are expressed as Evans blue in $\mathrm{ng} / \mathrm{mg}$ tissue.

Measurement of intracellular ROS production by flow cytometry. BMMCs were incubated with $1 \mu \mathrm{g} / \mathrm{ml}$ anti-OVA IgE for 16 hours at $37^{\circ} \mathrm{C}$. Cells were then washed and incubated with $2 \mu \mathrm{M}$ CM-H2DCFDA (ThermoFisher) in Tyrode's buffer for 30 minutes at $37^{\circ} \mathrm{C}$. Cells were washed and resuspended in Tyrode's buffer and challenged with OVA $(10 \mu \mathrm{g} / \mathrm{ml})$ at different times. Cell fluorescence was analyzed $(10,000$ events) using flow cytometry.

$\left[\mathrm{Ca}^{2+}\right]$ i measurement. Sensitized and challenged mast cells were loaded with $2.5 \mu \mathrm{M}$ of Fluo-4-acetomethoxy ester (ThermoFisher) for 1 hour in the dark at room temperature. After washing, cells were challenged with $10 \mu \mathrm{g} / \mathrm{ml}$ OVA and imaged at $488 \mathrm{~nm}$ excitation to detect intracellular free calcium continuously for 90 to 120 seconds. Each experiment was done at least three 3, and at least 100 cells were analyzed each time. 
In vivo BMMC injection. C57BL/6J or MMVV $\delta$ BMMCs $\left(1 \times 10^{7} / 200 \mu \mathrm{PBS}\right)$ were injected into CRE-sensitized MMVV $\delta$ mice through the tail vein 1 day before challenge (day 9 ). After 4 days of consecutive CRE challenge, mice were sacrificed on day 14, and lung tissues were harvested for the analysis of lung inflammation as described above.

Statistics. Data are presented as mean \pm SEM. The significance of differences among groups was determined by 1-way ANOVA (nonparametric test) using GraphPad Prism statistical software program (GraphPad Inc.). When 2 groups were compared, an unpaired, 2-tailed Student's $t$ test was used. A $P$ value of less than 0.05 was considered statistically significant.

Study approval. All animals studies presented in these studies were reviewed and approved by the Johns Hopkins University Animal Care and Use Committee.

\section{Author contribution}

JQ, DCD, YZ, and EL performed experiments, data analysis, and wrote the manuscript. WM supervised the tests for mouse lung function. MEA and PG designed and supervised the study and wrote the manuscript.

\section{Acknowledgments}

We thank Ho Man Tang and Ho Lam Tang for help with confocal microscopy. This work was supported by grants from the US NIH (R01ES021739, R21AI109062, and R21 AI121768 to PG; R01HL113001, R01HL079031, R01HL070250, and R01HL096652 to MEA; and P01 HL-10342 to WM); NSFC 81671561 and MOST 2016 YFC1305102 to ZY.

Address correspondence to: Peisong Gao, The Johns Hopkins Asthma \& Allergy Center, 5501 Hopkins Bayview Circle, Room 3B.71, Baltimore, Maryland 21224, USA. Phone: 410.550.2124; E-mail: pgao1@jhmi.edu.

1. Jarjour NN, Calhoun WJ. Enhanced production of oxygen radicals in asthma. J Lab Clin Med. 1994;123(1):131-136.

2. Abdala-Valencia $\mathrm{H}$, et al. Nonhematopoietic NADPH oxidase regulation of lung eosinophilia and airway hyperresponsiveness in experimentally induced asthma. Am J Physiol Lung Cell Mol Physiol. 2007;292(5):L1111-L1125.

3. Bowler RP. Oxidative stress in the pathogenesis of asthma. Curr Allergy Asthma Rep. 2004;4(2):116-122.

4. Li YJ, Takizawa H, Kawada T. Role of oxidative stresses induced by diesel exhaust particles in airway inflammation, allergy and asthma: their potential as a target of chemoprevention. Inflamm Allergy Drug Targets. 2010;9(4):300-305.

5. Al-Harbi NO, et al. Airway oxidative stress causes vascular and hepatic inflammation via upregulation of IL-17A in a murine model of allergic asthma. Int Immunopharmacol. 2016;34:173-182.

6. Ghio AJ, Carraway MS, Madden MC. Composition of air pollution particles and oxidative stress in cells, tissues, and living systems. J Toxicol Environ Health B Crit Rev. 2012;15(1):1-21.

7. Shalaby $\mathrm{KH}$, et al. Inhaled birch pollen extract induces airway hyperresponsiveness via oxidative stress but independently of pollen-intrinsic NADPH oxidase activity, or the TLR4-TRIF pathway. J Immunol. 2013;191(2):922-933.

8. Chan TK, et al. House dust mite-induced asthma causes oxidative damage and DNA double-strand breaks in the lungs. $J$ Allergy Clin Immunol. 2016;138(1):84-96.e1.

9. Anderson ME. Oxidant stress promotes disease by activating CaMKII. J Mol Cell Cardiol. 2015;89(Pt B):160-167.

10. Tombes RM, Faison MO, Turbeville JM. Organization and evolution of multifunctional $\mathrm{Ca}(2+) / \mathrm{CaM}$-dependent protein kinase genes. Gene. 2003;322:17-31.

11. Spinelli AM, et al. Smooth muscle CaMKII $\delta$ promotes allergen-induced airway hyperresponsiveness and inflammation. Pflugers Arch. 2015;467(12):2541-2554.

12. Erickson JR, et al. A dynamic pathway for calcium-independent activation of CaMKII by methionine oxidation. Cell. 2008;133(3):462-474

13. Luczak ED, Anderson ME. CaMKII oxidative activation and the pathogenesis of cardiac disease. J Mol Cell Cardiol. 2014;73:112-116.

14. He BJ, et al. Oxidation of CaMKII determines the cardiotoxic effects of aldosterone. Nat Med. 2011;17(12):1610-1618.

15. Luo M, et al. Diabetes increases mortality after myocardial infarction by oxidizing CaMKII. J Clin Invest. 2013;123(3):1262-1274

16. Hart PC, et al. MnSOD upregulation sustains the Warburg effect via mitochondrial ROS and AMPK-dependent signalling in cancer. Nat Commun. 2015;6:6053.

17. Purohit A, et al. Oxidized $\mathrm{Ca}(2+) /$ calmodulin-dependent protein kinase II triggers atrial fibrillation. Circulation. 2013;128(16):1748-1757.

18. Sanders PN, et al. CaMKII is essential for the proasthmatic effects of oxidation. Sci Transl Med. 2013;5(195):195ra97.

19. Singh MV, et al. Ca2+/calmodulin-dependent kinase II triggers cell membrane injury by inducing complement factor B gene expression in the mouse heart. J Clin Invest. 2009;119(4):986-996.

20. Li W, et al. The multifunctional Ca2+/calmodulin-dependent kinase II delta (CaMKIIdelta) controls neointima formation after carotid ligation and vascular smooth muscle cell proliferation through cell cycle regulation by p21. J Biol Chem. 2011;286(10):7990-7999.

21. Koval OM, et al. CaV1.2 beta-subunit coordinates CaMKII-triggered cardiomyocyte death and afterdepolarizations. Proc Natl 
Acad Sci USA. 2010;107(11):4996-5000.

22. Amin K. The role of mast cells in allergic inflammation. Respir Med. 2012;106(1):9-14

23. Galli SJ, Grimbaldeston M, Tsai M. Immunomodulatory mast cells: negative, as well as positive, regulators of immunity. Nat Rev Immunol. 2008;8(6):478-486.

24. Kalesnikoff J, Galli SJ. New developments in mast cell biology. Nat Immunol. 2008;9(11):1215-1223.

25. Schäfer B, et al. Mast cell anaphylatoxin receptor expression can enhance IgE-dependent skin inflammation in mice. $J$ Allergy Clin Immunol. 2013;131(2):541-8.e1.

26. Zhou Y, et al. Aryl hydrocarbon receptor controls murine mast cell homeostasis. Blood. 2013;121(16):3195-3204.

27. Sibilano R, Pucillo CE, Gri G. Allergic responses and aryl hydrocarbon receptor novel pathway of mast cell activation. Mol Immunol. 2015;63(1):69-73.

28. Morita $\mathrm{H}$, et al. An interleukin-33-mast cell-interleukin-2 axis suppresses papain-induced allergic inflammation by promoting regulatory T cell numbers. Immunity. 2015;43(1):175-186.

29. Di Stefano A, et al. Mast cells in the airway mucosa and rapid development of occupational asthma induced by toluene diisocyanate. Am Rev Respir Dis. 1993;147(4):1005-1009.

30. Kaur D, et al. IL-33 drives airway hyper-responsiveness through IL-13-mediated mast cell: airway smooth muscle crosstalk. Allergy. 2015;70(5):556-567.

31. Lewis RJ, Chachi L, Newby C, Amrani Y, Bradding P. Bidirectional counterregulation of human lung mast cell and airway smooth muscle $\beta 2$ adrenoceptors. J Immunol. 2016;196(1):55-63.

32. Juncadella IJ, et al. Apoptotic cell clearance by bronchial epithelial cells critically influences airway inflammation. Nature. 2013;493(7433):547-551.

33. Xu T, et al. Aryl hydrocarbon receptor protects lungs from cockroach allergen-induced inflammation by modulating mesenchymal stem cells. J Immunol. 2015;195(12):5539-5550

34. Bradding P, Walls AF, Holgate ST. The role of the mast cell in the pathophysiology of asthma. J Allergy Clin Immunol. 2006;117(6):1277-1284.

35. Andersson C, Tufvesson E, Diamant Z, Bjermer L. Revisiting the role of the mast cell in asthma. Curr Opin Pulm Med. 2016;22(1):10-17.

36. Sumi M, et al. The newly synthesized selective $\mathrm{Ca} 2+/$ calmodulin dependent protein kinase II inhibitor $\mathrm{KN}-93$ reduces dopamine contents in PC12h cells. Biochem Biophys Res Commun. 1991;181(3):968-975.

37. Gao P, et al. Functional effects of TGF- $\beta 1$ on mesenchymal stem cell mobilization in cockroach allergen-induced asthma. $J$ Immunol. 2014;192(10):4560-4570.

38. Jaffer OA, et al. Mitochondrial-targeted antioxidant therapy decreases transforming growth factor- $\beta$-mediated collagen production in a murine asthma model. Am J Respir Cell Mol Biol. 2015;52(1):106-115.

39. Rosenstreich DL, et al. The role of cockroach allergy and exposure to cockroach allergen in causing morbidity among inner-city children with asthma. NEngl J Med. 1997;336(19):1356-1363.

40. Gruchalla RS, et al. Inner City Asthma Study: relationships among sensitivity, allergen exposure, and asthma morbidity. $J$ Allergy Clin Immunol. 2005;115(3):478-485.

41. Togias A, Fenton MJ, Gergen PJ, Rotrosen D, Fauci AS. Asthma in the inner city: the perspective of the National Institute of Allergy and Infectious Diseases. J Allergy Clin Immunol. 2010;125(3):540-544.

42. Sohn MH, Kim KE. The cockroach and allergic diseases. Allergy Asthma Immunol Res. 2012;4(5):264-269.

43. Bacsi A, Pan L, Ba X, Boldogh I. Pathophysiology of bronchoconstriction: role of oxidatively damaged DNA repair. Curr Opin Allergy Clin Immunol. 2016;16(1):59-67.

44. Singh MV, Swaminathan PD, Luczak ED, Kutschke W, Weiss RM, Anderson ME. MyD88 mediated inflammatory signaling leads to CaMKII oxidation, cardiac hypertrophy and death after myocardial infarction. J Mol Cell Cardiol. 2012;52(5):1135-1144.

45. Swaminathan PD, et al. Oxidized CaMKII causes cardiac sinus node dysfunction in mice. J Clin Invest. 2011;121(8):3277-3288.

46. Viatchenko-Karpinski S, et al. Intracellular Na+ overload causes oxidation of CaMKII and leads to Ca2+ mishandling in isolated ventricular myocytes. J Mol Cell Cardiol. 2014;76:247-256.

47. Nadeem A, Siddiqui N, Al-Harbi NO, Al-Harbi MM, Ahmad SF. TLR-7 agonist attenuates airway reactivity and inflammation through Nrf2-mediated antioxidant protection in a murine model of allergic asthma. Int J Biochem Cell Biol. 2016;73:53-62.

48. Nadeem A, Alharbi NO, Vliagoftis H, Tyagi M, Ahmad SF, Sayed-Ahmed MM. Proteinase activated receptor-2-mediated dual oxidase-2 up-regulation is involved in enhanced airway reactivity and inflammation in a mouse model of allergic asthma. Immunology. 2015;145(3):391-403.

49. Chiba T, Uchi H, Tsuji G, Gondo H, Moroi Y, Furue M. Arylhydrocarbon receptor (AhR) activation in airway epithelial cells induces MUC5AC via reactive oxygen species (ROS) production. Pulm Pharmacol Ther. 2011;24(1):133-140.

50. Moura-Alves $\mathrm{P}$, et al. AhR sensing of bacterial pigments regulates antibacterial defence. Nature. 2014;512(7515):387-392.

51. Bessede A, et al. Aryl hydrocarbon receptor control of a disease tolerance defence pathway. Nature. 2014;511(7508):184-190.

52. Lambeth JD. NOX enzymes and the biology of reactive oxygen. Nat Rev Immunol. 2004;4(3):181-189.

53. Liu X, et al. Induction of mast cell accumulation by tryptase via a protease activated receptor- 2 and ICAM-1 dependent mechanism. Mediators Inflamm. 2016;2016:6431574.

54. Taub D, et al. Bone marrow-derived murine mast cells migrate, but do not degranulate, in response to chemokines. J Immunol. 1995;154(5):2393-2402.

55. Juremalm M, Olsson N, Nilsson G. Selective CCL5/RANTES-induced mast cell migration through interactions with chemokine receptors CCR1 and CCR4. Biochem Biophys Res Commun. 2002;297(3):480-485.

56. Nilsson G, Mikovits JA, Metcalfe DD, Taub DD. Mast cell migratory response to interleukin- 8 is mediated through interaction with chemokine receptor CXCR2/Interleukin-8RB. Blood. 1999;93(9):2791-2797.

57. McCurdy JD, Olynych TJ, Maher LH, Marshall JS. Cutting edge: distinct Toll-like receptor 2 activators selectively induce different classes of mediator production from human mast cells. J Immunol. 2003;170(4):1625-1629.

58. Supajatura V, Ushio H, Nakao A, Okumura K, Ra C, Ogawa H. Protective roles of mast cells against enterobacterial infection are mediated by Toll-like receptor 4. J Immunol. 2001;167(4):2250-2256. 
59. McNeil BD, et al. Identification of a mast-cell-specific receptor crucial for pseudo-allergic drug reactions. Nature.

2015;519(7542):237-241.

60. Moskovitz J, Bar-Noy S, Williams WM, Requena J, Berlett BS, Stadtman ER. Methionine sulfoxide reductase (MsrA) is a regulator of antioxidant defense and lifespan in mammals. Proc Natl Acad Sci USA. 2001;98(23):12920-12925.

61. Wilhelms $\mathrm{OH}$. An improved automated fluorimetric method for determination of histamine. J Immunol Methods. $1980 ; 36(3-4): 221-226$. 